LA-UR- $9 R-22 \% 2$

Approved for public release; distribution is unlimited.

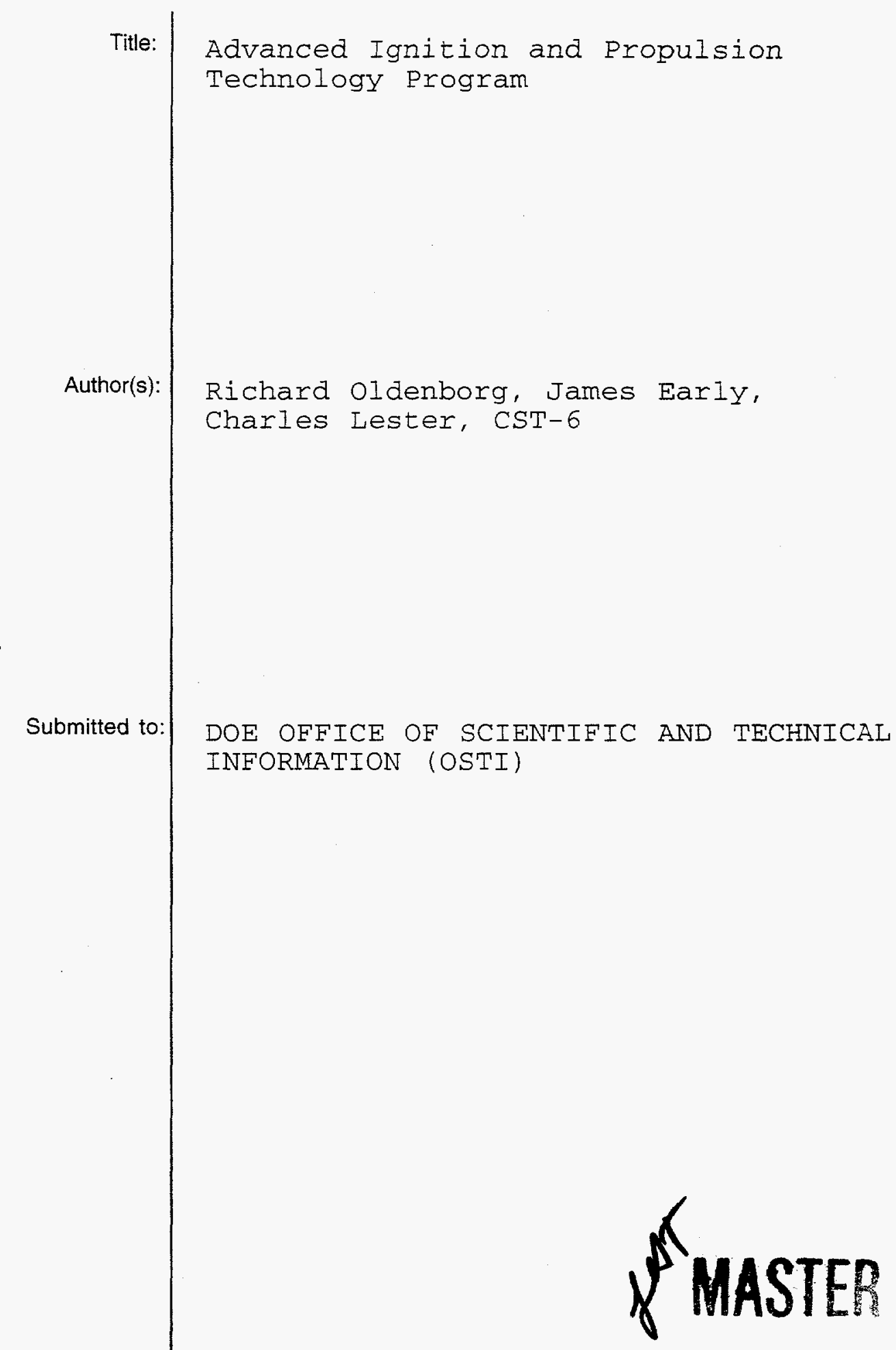

\section{Los Alamos}

NATIONAL LABORATORY

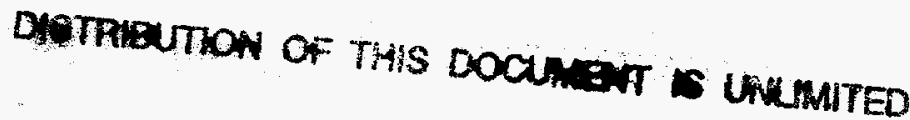

Los Alamos National Laboratory, an affirmative action/equal opportunity employer, is operated by the University of California for the U.S. Department of Energy under contract W-7405-ENG-36. By acceptance of this article, the publisher recognizes that the U.S. Government retains a nonexclusive, royalty-free license to publish or reproduce the published form of this contribution, or to allow others to do so, for U.S. Government purposes. Los Alamos National Laboratory requests that the publisher identify this article as work pertormed under the auspices of the U.S. Department of Energy. The Los Alamos National Laboratory strongly supports academic freedom and a researcher's right to publish; as an institution, however, the Laboratory does not endorse the viewpoint of a publication or guarantee its technical correctness. 


\section{DISCLAIMER}

This report was prepared as an account of work sponsored by an agency of the United States Government. Neither the United States Government nor any agency thereof, nor any of their employees, makes any warranty, express or implied, or assumes any legal liability or responsibility for the accuracy, completeness, or usefulness of any information, apparatus, product, or process disclosed, or represents that its use would not infringe privately owned rights. Reference herein to any specific commercial product, process, or service by trade name, trademark, manufacturer, or otherwise does not necessarily constitute or imply its endorsement, recommendation, or favoring by the United States Government or any agency thereof. The views and opinions of authors expressed herein do not necessarily state or reflect those of the United States Government or any agency thereof. 


\section{DISCLAIMER}

Portions of this document may be illegible in electronic image products. Images are produced from the best available original document. 


\title{
Advanced Ignition and Propulsion Technology Program
}

\author{
Richard Oldenborg*, James Early, and Charles Lester
}

\begin{abstract}
This is the final report of a three-year, Laboratory Directed Research and Development (LDRD) project at the Los Alamos National Laboratory (LANL). Reliable engine re-ignition plays a crucial role in enabling commercial and military aircraft to fly safely at high altitudes. This project addressed research elements critical to the optimization of laser-induced fuel ignition and the development of a realistically deployable laser-based igniter. The effort initially involved a collaborative research and development agreement with B. F. Goodrich Aerospace and Laser Fare, Inc. The work involved integrated experiments with theoretical modeling to provide a basic understanding of the chemistry and physics controlling the laser-induced ignition of fuel aerosols produced by turbojet engine injectors. In addition, we defined advanced laser igniter configurations that minimize laser packaging size, weight, complexity and power consumption. These innovative ignition concepts were shown to reliably ignite jet fuel aerosols over a broad range of fuel/air mixture and at fuel temperatures as low as $-40 \operatorname{deg} \mathrm{F}$. The demonstrated fuel ignition performance was highly superior to that obtained by the state-of-the-art, laser-spark ignition method utilizing comparable laser energy. We also developed a laser-based method that effectively removes optically opaque deposits of fuel hydrocarbon combustion residues from laser window surfaces. Seven patents have been either issued or are pending that resulted from the technology developments within this project.
\end{abstract}

\section{Background and Research Objectives}

Conventional commercial and military turbo-jet aircraft engines use capacitive discharge ignition systems to initiate fuel combustion. The fuel-rich conditions required to ensure engine re-ignition during flight yield less than optimal engine performance, which in turn reduces fuel economy and generates considerable pollution in the exhaust. Microwave- and laser-based approaches are possible alternatives to capacitive discharge. The microwave approaches involve modeling and, if necessary, redesigning a combustor shape to form a low-Q microwave cavity, which will ensure microwave breakdown of the air/fuel mixture just ahead of the nozzle with or without a catalyst coating. These approaches also involve conducting radio-frequency (RF) heating of ceramic elements

\footnotetext{
*Principal Investigator, e-mail: ro@lanl.gov
} 
that have large loss tangents. The laser-based approaches are fuel ignition via laser-spark breakdown and via photo-dissociation of fuel hydrocarbons and oxygen. Replacing conventional systems with either of these two new systems will yield combustion in leaner jet fuel/air mixtures. As a result, the aircraft would operate at high altitudes with lower engine maintenance and significantly higher fuel economy.

Investigation of these new approaches involves Iaser system design and key window studies; ignition studies on gas-phase fuel-air mixtures and aerosols: microwave modeling including RF breakdown in a combustor; developing appropriate ceramics; and working with industry to develop this technology. This technology requires expertise presently not found in the private sector, which presently manufactures all igniters for military and commercial use. Los Alamos has the resources and multidisciplinary staff to develop these technologies in partnership with one or more private companies. The primary objective of this project was to establish a proof of principle for either a microwave- or laser-based system that enables aircraft to effectively function at high altitudes.

\section{Importance to LANL's Science and Technology Base and National R\&D Needs}

Although the primary goal of this project has been the development of an effective, energy efficient and deployable fuel ignition system for both commercial and military turbojet engine applications, tangible payoffs to the DOE and LANL's technology base exist within alternative uses for the ignition technology developed. Spin-off applications that support LANL's primary mission within the DOE complex include enhanced initiation of explosive and pyrotechnic materials, as well as pollution reduction issues. Developments in the first area may significantly contribute to nuclear weapons stockpile safeguards and quality assurance issues. In the second area, experiments conducted by other efforts have shown considerable potential for our ignition technology to stabilize the ultra-lean combustion of fuel in power generating turbines, providing potentially significant reductions in NOX emissions. Discussions with leaders in the power utility and the turbine manufacturing industries indicate that future developments in this application area will have global impact, permitting both modern and older power plants to operate more cleanly. 


\section{Accomplishments}

In the initial part of this project, we participated in a Collaborative Research and Development Agreement (CRADA) with B. F. Goodrich Aerospace and Laser Fare, Inc. The primary goal of this collaboration was to develop an advanced ignition system for aircraft turbo-jet engines that could replace the currently used capacitive discharge igniters. We investigated two technology paths to this goal incorporating both laserassisted and microwave-based ignition approaches. The results of the CRADA effort are described within a final report (LA-CRADA-97-0002) submitted to the DOE at the close of the CRADA portion of the project in FY 1996. A brief summary of the CRADA results is given below.

The laser ignition portion of the CRADA effort resulted in the development and evaluation of two novel, laser-induced, jet-fuel-ignition concepts. These concepts utilized multiple laser wavelengths and pulse formats to enhance fuel ignition performance. The first of these novel concepts, the dual-laser-pulse (DLP) ignition method, utilized the laser light pumping of a previously established laser spark to actively heat the fuel medium for durations of time much longer than can be readily obtained by a single laser pulse alone. A second ignition concept, called the laser preheat ignition method, utilized the inherent light-absorptive property of the fuel at a 3-micron wavelength to vaporize aerosol fuel droplets prior to gas-phase ignition via a laserinduced spark.

The ignition of Jet A fuel sprays generated by a commercial air-blast-type fuel atomizer, operated in free-air under ambient conditions, was investigated. Direct comparison of jet-fuel-spray ignition performance relative to that obtained by a more conventional laser-spark approach that utilizes a SLP was experimentally evaluated as a function of laser and fuel spray parameters. Significant improvements in fuel ignition performance were obtained, which include lower laser energy requirements for consistent fuel ignition and lesser ignition sensitivity to fuel composition variations. Another portion of the CRADA effort addressed window contamination issues. A laser ablation method, capable of removing fuel residues from optical surfaces, was developed and evaluated experimentally. Three pending patents resulted from the CRADA effort.

Following termination of the CRADA effort, our effort was redirected toward fuel ignition interests within the DoD. The R\&D objectives, experimental approaches and results obtained from this part of the project are the subject of the remainder of this report. Areas of endeavor included 1) the ignition of fuel under ultra-cold conditions, 2) a demonstration of developed ignition technology under actual turbine engine operating conditions, 3) the development of deployable laser igniter hardware concepts that 
enhance cost effectiveness, durability and packaging size, and 4) the presentation and discussion of our laser-induced ignition technology with numerous potential sponsors within the military [Army (TACOM and ARL), Air Force (Wright-Patterson) and Navy (NAWC)], DOE (PETC and METC), and manufacturers and users of turbine and internal combustion engines (Caterpillar Tractor/Solar Turbines and Allied Signal).

The experimental results obtained from the R\&D program demonstrated the ability of our multiple-laser-pulse (MLP) technology to provide consistent ignition of jet fuel sprays over a wide range of fuel temperature and fuel flow conditions. Direct comparison of the MLP ignition results with that obtained by a more conventional SLP ignition approach under identical fuel/air flow conditions has shown the considerable benefits provided by the MLP approach with respect to lesser sensitivity to fuel composition variations and lower laser energy requirements for reliable fuel spray ignition.

Ignition performance for both the MLP and SLP approaches were evaluated through laboratory-scale investigations of fuel spray ignition, as well as investigations conducted on a combustor test rig operated under realistic turbine engine fuel and air flow rates. The results of these studies have defined the optimal operating parameters of a MLP igniter required to achieve reliable ignition of fuel sprays with respect to laser energy, temporal delay between laser pulses and initiation location within the fuel spray for a wide range of fuel parameters. In addition, compact, durable and energy-efficient laser hardware concepts were developed that enhance the deployment of a practical MLP igniter for aircraft and ground-based vehicles and power generation equipment.

During the course of this project, we have made many important contacts with potential collaborators and sponsors within the government, military and private industry sectors. In collaboration with CFD Research Corp., we are currently pursuing two SBIR programs funded by the Army to investigate the ignition of diesel and jet fuels under ultra-cold conditions for military diesel and turboshaft-engine applications. Another area of application for our ignition technology is the initiation of explosives and pyrotechnics, and several weapons programs at Los Alamos have implemented various aspects of the technologies developed within our fuel ignition program. Negotiations with DoD organizations (Edwards Air Force Base and DARPA) and private industry (Aerojet General, Solar Turbines and PNM- a power generation utility ) are currently being pursed to investigate the fuel-ignition and flame-stabilization capabilities of our laser-assisted ignition technology. The proposed experimental programs represent a broad range of laser-assisted ignition application areas, including the stabilization of ultra-lean 
combustion in gas turbines for pollution reduction, rocket thruster and main rocket propulsion engine ignition, and scramjet engine virtual flame-holding functions.

\section{Scientific Approach}

The research conducted after the end of the CRADA phase of the project was primarily to answer questions of significant importance to potential users of our laserinduced ignition technology. Some of these questions are: 1) Can our MLP ignition approach ignite jet fuel under ultracold turbine engine startup conditions?,2) What is the performance of our ignition approach under fuel and air flow conditions realized within an actual turbine engine?, 3) How does its performance in the above areas compare to the conventional SLP ignition approach--are there distinct advantages?, and 4) Can compact, durable and cost-effective laser hardware be developed to implement this ignition technology in aircraft? The following sections describe the experimental approaches taken to answer these questions and the results obtained.

\section{Dual-Laser-Pulse (DLP) Ignition Concept}

This laser-based fuel-ignition method was the first of three novel ignition concepts investigated, and is the subject of the experimental investigations described in the remainder of this report. The DLP ignition concept is fundamentally different with respect to the conventional laser-spark-ignition method and provides several distinct advantages. Of primary importance is the effective decoupling of breakdown plasma generation from the laser heating of the fuel medium. Initially a short duration, highpeak-power laser pulse is applied to the fuel medium to initiate a laser-light-absorptive breakdown plasma. This is then followed by the application of a second laser-light pulse with a longer duration and lower peak power that efficiently couples laser light energy into the previously established breakdown plasma and sustains the plasma over time durations typically exceeding the temporal length of the second laser pulse. By employing two laser pulses with differing temporal pulse length and peak power, the rate of energy delivery to the fuel/oxidizer medium is reduced while the duration over which the fuel medium is actively heated by the laser light is substantially increased.

\section{Laser Ignition Experimental Apparatus}

\section{Dual-Pulse Laser Instrumentation}

The laser apparatus used to provide light radiation with the required dual-pulse format is shown in Figure1. Two separate lasers were used for this task in the laboratory investigation, which allowed convenient adjustment of individual laser parameters such as pulse energy, pulse width, and an adjustable delay between the firing of the lasers. One laser produces short-duration, high-peak-power pulses, while the other produces 
longer, lower-peak-power laser light. The output of each laser was directed onto a dichromatic beam combiner, which permitted the spatial overlap and coaxial propagation of the two laser beams to a common focusing lens. When focused using a short focallength (FL) lens, a laser-induced breakdown spark is produced with the desirable fuel igniting properties described above.

The short duration pulses, 12 ns at full-width and half-maximum (FWHM), were provided by a Q-switched Nd:YAG laser operating at a wavelength of $1064 \mathrm{~nm}$. This laser could provide a maximum pulse energy of $550 \mathrm{~mJ}$. although the laser was typically operated at $80 \mathrm{~mJ} /$ pulse, which provided sufficiently high peak power to cause air breakdown when its output was focused by a short FL $(10 \mathrm{~cm})$ lens.

The longer duration laser pulse was obtained from a Q-switched Cr:LiSAF (chromium doped: lithium-strontium-aluminum-fluoride) laser. This wavelength-tunable laser was operated at a wavelength of $850 \mathrm{~nm}$ and provided an output pulse with a temporal length of typically $85 \mathrm{~ns}$ (FWHM) and a maximum pulse energy of $450 \mathrm{~mJ}$. This laser was nominally operated at a pulse energy of $350 \mathrm{~mJ}$. To attenuate the output of this laser as required for most of the experiments performed, a neutral density filter or rotatable polarizer or both were used. This was done to prevent laser-pulse energydependent changes in pulse length from occurring, as would be the case if laser pulse energy were to be reduced by adjusting laser pump energy. All energy values reported were measured after laser light passage through attenuators and the dichromatic beam combiner, but do not include the small 5.0\% Fresnel reflection losses resulting from the uncoated silica focusing lens. Both lasers were operated with a multiple transverse mode in their output. All laser light was contained in a horizontal plane.

For those experiments that utilized the Q-switched Nd:YAG output alone, the Cr:LiSAF laser was turned off or blocked so that only the Nd:YAG output would pass through the beam combiner to the focusing lens. The output pulse energy of this laser was typically adjusted by pump energy to the flashlamps, since no significant alteration of pulse length was observed over the full range of output energy.

Following the beam combiner, the laser light was propagated over a distance of about $3 \mathrm{~m}$ to a short-FL(typically $10 \mathrm{~cm}$ ) lens, which was located near the fuel spray enclosure. This lens provided sufficient light power density at the focal plane to produce an air breakdown spark and also projected the igniting laser spark at a sufficient distance from the lens to prevent fouling of the lens surface by fuel droplets from the fuel spray region. Laser spot diameters in the focal plane of the $10-\mathrm{cm}$ FL lens were measured by a scanning optical fiber method to be 65 microns (FWHM) for the Nd:YAG light and 75 microns (FWHM) for the Cr:LiSAF radiation. 


\section{Fuel Injector Operation and Characterization}

Two types of fuel-spray injection nozzles were used in these investigations: a duplex and an air-blast-type fuel atomizer. Most of the work was conducted using a prototype air-blast nozzle developed by CFD Research Corp. for the U.S. Air Force's JTAGG advanced turbine engine development program. This nozzle has a flow number of 4.4 and was operated routinely at a fuel differential pressure of 8.5 psia and air flow differential pressure of 5.0 inch $\mathrm{H}_{2} 0$. At these pressure settings, the injector equivalence ratio for fuel and air flows was about 0.95 . Transducer-type pressure gauges were used to monitor fuel and air pressures directly at the fuel nozzle. Pressure measurement accuracies of $0.02 \mathrm{psi}$ and 0.01 -inch $\mathrm{H}_{2} \mathrm{O}$, respectively, were obtained. Fuel was fed from a pressurized fuel vessel while the air flow was provided by a centrifugal pump. Using this type of fuel delivery, the fuel flow could be accurately regulated to $+/-0.1 \mathrm{psi}$. Air flow could be regulated to $+/$ - 0.2 -inch $\mathrm{H}_{2} \mathrm{O}$.

Cooling of fuel and air flows was achieved by installation of heat exchangers in the fuel feed vessel and also in the fuel and air feed lines to the nozzle. A $1-\mathrm{kW}$ cryogenic recirculating cooler was used as the cooling source. Using this instrumentation the fuel could be cooled to a minimum temperature of $-43 \mathrm{deg} F$ as measured at the fuel injector nozzle. Although the cooling system was capable of cooling the fuel to even lower temperatures, geling of the fuel prevented consistent flow to the injector. Cooling of the air was limited to a minimum temperature of about $10 \mathrm{deg} F$ due to exchanger inefficiencies at this air flow rate. For most experiments performed, the fuel temperature was adjusted over a -43 to 75 deg $F$ temperature range, while the air flow through the nozzle was maintained at ambient temperature $(75 \mathrm{deg} F)$.

A laser-based Doppler PDPA (particle distribution profile analysis) measurement of the fuel spray parameters was performed to characterize the distributions of fuel droplet size, number density, velocity, and volume flux $\left(\mathrm{scc} / \mathrm{s}-\mathrm{cm}^{2}\right)$ within the fuel spray as a function of distance from the nozzle and radial distance from the nozzle axis. Measurements of these fuel cloud parameters were performed at ambient temperature ( 20 $\operatorname{deg} \mathrm{C})$ and at a reduced fuel temperature $(-5 \mathrm{deg} C)$ and at fuel and air flow differential pressures of 8.5 psia and 5.0-inch $\mathrm{H}_{2} \mathrm{O}$, respectively. Air temperature was maintained at $20 \mathrm{deg}$ C. An example of the information obtained is shown in Figures $2 \mathrm{a}$ through $2 \mathrm{~d}$. The volume flux distribution (Figure $2 \mathrm{a}$ ) for $-5 \mathrm{deg} \mathrm{C}$ fuel is plotted as a function of radial distance from the nozzle axis for several longitudinal distances from the nozzle face. Similar measurements of number density, particle size and axial velocity were also performed and are shown in Figures $2 b$ through $2 d$ for fuel cooled to a temperature of -5 $\operatorname{deg} \mathrm{C}$. These measurements provided information critical to the analysis of the laser 
ignition performance relative to the local fuel composition at the site of laser spark initiation.

\section{Reduced-Fuel-Temperature Ignition Experiments}

The reduced-temperature fuel-ignition experiments performed were conducted utilizing both the DLP and SLP ignition approaches. The initial experiments performed utilized only the SLP approach to define 1) the performance of the conventional SLP method to initiate cold, low-volatility fuel, 2) to map the areas of the fuel cloud that provide the highest probability for laser-spark-induced ignition. The remaining portions of the experimental effort characterized the performance of the DLP fuel ignition method. Typically, the DLP experimental results are accompanied by results achieved utilizing the SLP ignition approach so that a direct comparison of ignition performance under identical fuel parameter conditions can be made.

Most of the fuel ignition experiments utilized fuel sprays produced by an air-blasttype atomizer supplied by CFD Research Corp., although in some experiments a duplex, pressurized, fuel-spray-type atomizer was used prior to the delivery of the air-blast injector. Unlike the air-blast atomizer, the fuel spray from this nozzle was not calibrated by PDPA analysis such as that reported above for the air-blast model, although some measurements characterizing fuel flow within specific regions of the fuel cloud were performed. A description of this measurement method is provided within experiment \# 5, where this information was utilized.

For all of the fuel ignition experiments, the injector nozzle was operated in free air at ambient air temperature ( $20 \mathrm{deg} C$ ) and pressure (580 torr), with no combustion chamber-like enclosure surrounding the injector. The injector was positioned at one end of a large ventilated box ( 3 - $\mathrm{ft}$ high $\mathrm{x} 2-\mathrm{ft}$ wide $\mathrm{x} 5$ - $\mathrm{ft}$ long ), used to contain combustion products and unburned fuel. The fuel injector was positioned so that the axis of the fuel spray would be contained within the horizontal plane. Fuel-igniting laser light was also directed horizontally such that perpendicular incidence to the axis of the conical fuel cloud would be obtained, as shown in Figure 3. A 10-cm FL lens was then inserted in the laser output beam at a distance of about $10 \mathrm{~cm}$ from the nozzle axis. In order to investigate initiation at various regions of the fuel cloud, the fuel nozzle was mounted on an $\mathrm{X}-\mathrm{Y}-\mathrm{Z}$ translator so that virtually any position within the fuel cloud could be addressed by the laser spark by moving the fuel spray relative to the spatially fixed laser spark. A video camera located above the fuel plume is used to record the ignition experiments for off-line analysis. 


\section{SLP Fuel Ignition Experiments}

Experiment \#1 - Ignition Probability vs. Fuel Temperature. The first experiments to be performed that identified the sensitivity of the laser-spark ignition process to fuel temperature utilized a duplex-pressurized fuel atomizer of a type commonly used in commercial aircraft turbine engines. This nozzle was operated at a fuel differential pressure of 20.0 psig and an air pressure of 2.0 in. $\mathrm{H}_{2} \mathrm{O}$. No PDPA analysis of spray droplet distributions were conducted for this nozzle. For this nozzle air flow was not used to atomize fuel, but to keep the nozzle atomization ports from becoming clogged with combustion residues.

The experimental apparatus used is shown in Figure 3. The fuel spray was ignited using a laser spark produced by the light output of a Q-switched Nd:YAG laser operating at a wavelength of $1064 \mathrm{~nm}$. A 10-cm FL lens was used to focus the 12-ns duration pulses of the laser to a small spot size within the fuel cloud at a "sweet spot" for ignition. This "sweet spot" was located within the fuel spray at a position $1.0 \mathrm{~cm}$ from the nozzle and $8.75 \mathrm{~mm}$ from the nozzle axis. A smaller capacity Nd:YAG laser was used for this experiment that was operated at its maximum output pulse energy of $120 \mathrm{~mJ}$. Fuel at an ambient temperature of $85 \mathrm{deg} F$ was used during these measurements. An extensive example of the ignition mapping procedure is demonstrated in the next section, and was performed using an air-blast style of fuel atomizer utilized in the majority of the reducedtemperature experiments.

Following the determination of an appropriate location within the fuel spray to initiate combustion, the fuel was reduced in temperature from ambient to $-43 \mathrm{deg} F$. The results of this experiment are shown in Figure 4. Each data point within the plot represents the average ignition frequency resulting from twenty ignition trials obtained at the designated initiation location within the fuel cloud, over the $+85 \operatorname{deg} \mathrm{F}$ to $-43 \operatorname{deg} \mathrm{F}$ temperature range.

This experiment identified the difficulty of the conventional laser spark ignition approach to ignite colder and less volatile fuel. The results showed a dramatic decrease in laser induced ignition performance at fuel temperatures below $+45 \mathrm{deg} F$ for fixed laser pulse energy.

\section{Experiment \#2 - Ignition Mapping of the Air-blast Injector at Reduced Fuel}

Temperatures. In order to provide more relevance of ignition results to DoD issues, the following series of experiments utilized the prototype JTAGG air-blast nozzle to provide sprays of cooled fuel. Initially the ignition characteristics of the fuel cloud were mapped as a function of radial distance of the laser spark from the nozzle axis and as a function of distance from the nozzle face. This was done to identify the "sweet spots" for ignition 
within the fuel cloud that would support ignition. This information would be utilized in subsequent experiments. Furthermore, we wished to determine how these identified "sweet spots" correlate with the known fuel droplet distributions. The fuel injector nozzle supplied by CFD Research Corp. was characterized by the PDPA analysis described above.

A single Q-switched pulse from the Nd:YAG laser at a pulse energy of 200 $\mathrm{mJ} /$ pulse was used to map the ignition characteristics of the fuel cloud at a reduced fuel temperature $(-5 \operatorname{deg} C)$. This laser was considerably larger than that utilized in the pervious experiment and provided a maximum pulse energy of $550 \mathrm{~mJ}$ with a pulse duration of $10 \mathrm{~ns}$. Initializing the location of the laser spark was done by centering the laser spark upon the center of spray nozzle at a position directly above the nozzle face. Scanning of the nozzle was then done by stepping the $X-Y-Z$ motion translator, to which the nozzle is attached. The longitudinal distance from the nozzle was set, followed by sequential movement of the nozzle along a radius in increments of $1.25 \mathrm{~mm}$. Increments of the longitudinal motion along the nozzle axis was typically $1.0 \mathrm{~cm}$. The radial scans were always performed by moving the laser spark from the nozzle axis toward regions of the fuel plume located closer to the laser light focusing lens.

The results of this investigation are shown in Figure 5, in which ignition frequency is plotted versus the radial position of the laser spark for several longitudinal positions from the nozzle face. Ignition frequency is defined here as the fraction of successful laser-induced ignitions obtained to the total number of ignition attempts. The criteria for a successful ignition is initiated combustion that propagates throughout the fuel plume and is not confined to the immediate area of initiation. A video camera located above the fuel plume is used to record each ignition event for off-line analysis of the ignition frequency achieved. Each data point in Figure 5 corresponds to 20 laser shots directed at that particular location within the fuel spray.

This study showed that although none of the radial scans resulted in a $100 \%$ ignition probability for any given location within the cooled fuel cloud addressed by the laser spark, various local maxima were identified by the ignition maps obtained at each distance from the nozzle. These local maxima or "ignition sweet spots" apparently correlate well with local maxima in the volume flux, as noted from the PDPA data of Figure 2. In other words, the more fuel present to be ignited, the higher the ignition probability. This result may relate more closely to the probability of finding an ignitable fuel droplet within the laser heated volume at any given instant of time, since the radial ignition "sweet spot" locations also correlate well with the maximum in droplet density for any given distance from the nozzle. Similar results were obtained during the CRADA 
portion of this project characterizing the laser-induced ignition of jet fuel sprays at ambient temperature.

The broader width of the ignition profile compared to that of the volume flux distribution may reflect the finite width of the laser spark (about $0.3 \mathrm{~cm}$ ) and the volume of fuel/air heated (which is time dependent and not well defined). For example, at a laser spark location within the fuel cloud corresponding to a radial distance of $6.25 \mathrm{~mm}$ and a distance from the nozzle of $1.0 \mathrm{~cm}$, where according to the PDPA analysis of Figure 2 virtually no fuel droplets exist, there is a $10 \%$ probability of achieving ignition with each laser shot. Another potential explanation may be linked to low number densities of very fine and easily combustible fuel droplets carried to regions outside of the main flow regions of the fuel cloud due to eddy currents in the air flow. Such a situation is indicated in the on axis $(\mathrm{r}=0 \mathrm{~mm})$ ignition profile for $1 \mathrm{-cm}$ distance from the nozzle in which low frequency ignition is achieved. where again no substantial fuel flow exists. Nevertheless, the main objective of the measurement was achieved, i.e. the determination (or mapping) of "ignition sweet spots" within the fuel spray, information that was used in subsequent experiments.

Experiment \#3 - SLP Ignition Performance at Reduced Fuel Temperature. The next series of experiments defined the probability for the $\mathrm{Nd}$ :YAG-generated spark to induce the ignition of the fuel cooled to a temperature of -5 deg $\mathrm{C}$ as a function of Nd:YAG pulse energy applied and location within the fuel spray. For these experiments, the laser spark was sequentially directed to those "sweet spot" locations in the fuel cloud as identified in the previous experiment. These locations had the longitudinal and radial coordinates of $(1.0 \mathrm{~cm}, 12.5 \mathrm{~mm}),(2.0 \mathrm{~cm}, 16.5 \mathrm{~mm}),(3.0 \mathrm{~cm}, 20.0 \mathrm{~mm})$ and $(4.0 \mathrm{~cm}$, $25.0 \mathrm{~mm}$ ). The laser operated at pulse energies in the range of $0-550 \mathrm{~mJ}$. The data taking occurred in the following manner. Initially the laser spark would be located at one of the "sweet spots" indicated above, and the laser pulse energy set to some lower value in the range of 0-550 mJ. A laser spark would be induced within the fuel and a "go or no go" ignition result observed. An ignition frequency was then calculated based upon the number of successful ignitions achieved out of a total of 80 individual ignition attempts. The laser pulse energy was then increased to another value and the ignition frequency recorded. Typically the laser energy would be increased in increments of about $20 \mathrm{~mJ}$. When the ignition probability for the entire range of pulse energy was obtained, the laser spark would be positioned at the next "sweet spot" location within the fuel cloud and the whole data taking process repeated.

The result of this effort is shown in Figure 6, in which the ignition frequency is plotted vs. laser pulse energy for several locations within the fuel cloud. The data shows 
that the ignition frequency increases rather slowly as laser energy is increased and that the laser energy required to reliably ignite fuel at a reduced temperature of $-5 \mathrm{deg} F$ is rather large (at least $300 \mathrm{~mJ}$, depending upon the distance from the injector ). More interesting, the S-shaped curves obtained approach the $100 \%$ ignition nearly asymptotically, requiring a $33 \%$ increase in pulse energy to achieve the last $10 \%$ in ignition frequency at the best location within the fuel cloud. At greater distances from the nozzle the situation worsens, where the trailing of the ignition curve becomes more pronounced and the linear portions of the curves have a smaller slope. The question now arises: Can the DLP ignition approach, which has previously shown to provide greater ignition reliability and energy efficiency for fuel at ambient temperatures, also provide superior ignition performance at reduced temperature? This was investigated in the following experiment.

\section{DLP Ignition of Cold Jet Fuel}

The primary objectives of the cold fuel ignition experiments were 1) to define the ignition characteristics of the DLP approach, 2) to compare these results with those obtained utilizing the more conventional SLP approach, and 3) to identify any possible benefits provided by the application of the DLP ignition approach to cold jet fuel with respect to ignition reliability and energy efficiency.

Experiment \#4 -Temporal Delay Between Pulse Application. One of the first tasks required to appropriately apply DLP ignition was to determine the optimal temporal spacing between the application of first and second laser pulses within the dual pulse ignition format. From previous work, it was shown that the outcome of the dual pulse ignition process depends strongly upon efficient deposition of second pulse energy into the plasma generated by the first, higher peak power, laser pulse. The efficiency of the energy coupling process depends significantly upon the temporal delay between the establishment of the air breakdown plasma and its subsequent pumping by the second, longer-duration light pulse.

An initiation location within the fuel was chosen near one of the "sweet spots" determined by the SLP ignition mappings as described previously. The dual pulse igniting light is then applied to induce fuel ignition in a manner such that the firing of the two lasers was delayed by sequential larger values, typically starting at temporal coincidence. The firing of the second laser is delayed relative to the firing of the first laser pulse. Ignition frequency was recorded as a function of the inter-pulse timing delay for fixed first and second pulse energies. The particular pulse timing delay that results in a maximum ignition frequency was then chosen for the inter-pulse temporal spacing for the balance of the cold fuel ignition studies. An example of the result of this delay tuning 
procedure is given in Figure 7, where measured ignition frequency is plotted vs. the delay between the output of the laser pulses. This delay was measured as the time between the peak intensity points in the temporal profile of each pulse and was measured using two photodiodes sampling each laser output.

The data in Figure 7 was obtained for fixed first and second pulse energies of 120 and $240 \mathrm{~mJ}$, respectively, and for laser pulse widths of 12 and $85 \mathrm{~ns}$, respectively. Each data point within the plot represents the result of twenty ignition trials. As the inter-pulse delay was increased from zero to $75 \mathrm{~ns}$, the ignition frequency increased rapidly from a low value of about $35 \%$ to $100 \%$. Further increases in the delay produced no reduction in the fuel igniting characteristics of the combined laser pulses, until a delay of $175 \mathrm{~ns}$ was inserted. Beyond this value, the ignition frequency decreased slowly. The data indicates that the optimal ignition performance was achieved within a range of inter-pulse delays between 75 and $175 \mathrm{~ns}$. For the cooled fuel experiments, an optimal temporal delay of $100 \mathrm{~ns}$ was determined and used for virtually all subsequent dual pulse experiments.

Experiment \#5 - Ignition Sensitivity to Fuel Composition. The first DLP experiment in the cold jet fuel ignition portion of our work investigated the ability of the dual pulse ignition method to ignite various portions of the fuel cloud with widely varying composition. Identical experiments were also conducted utilizing the SLP ignition method to which DLP ignition performance could be compared. These experiments were conducted before receipt of the JTAGG nozzle and, in its place, a duplex, pressurized fuel nozzle was used. The nozzle was operated at a differential pressure of $20.0 \mathrm{psi}$ and at a fuel temperature of $-5 \mathrm{deg}$ C. Although no PDPA analysis of droplet distributions from this nozzle was performed, visual inspection indicated that the fuel droplets were considerably finer than those obtained from the air-blast JTAGG nozzle used in subsequent experiments were.

Ignition mapping identified the laser ignition "sweet spots" within the fuel cloud at longitudinal distances of 1.0, 2.0, 3.0, 4.0 and $5.0 \mathrm{~cm}$ from the nozzle face. The laser energy required for $100 \%$ consistent ignition was obtained for both the DLP and SLP ignition mechanisms. For the DLP case, the first pulse energy from the Nd:YAG laser was adjusted to $120 \mathrm{~mJ}$, and the second laser pulse energy was varied as required to obtain a $100 \%$ reliable ignition probability. The pulse widths of the first and second pulses were 12 and $86 \mathrm{~ns}$, respectively. For the SLP ignition case, the Q-switched $\mathrm{Nd}: Y A G$ was operated with a pulse length of $12 \mathrm{~ns}$ and at pulse energies to a maximum of $550 \mathrm{~mJ}$.

The experimental results, obtained for both the single and dual laser pulse ignition methods, are summarized in Figure 8, where the pulse energy required to achieve 
consistent ignition is plotted versus the volume flux measured at the ignition location within the fuel spray. Consistent ignition is defined here as twenty successful laserinduced ignitions of the fuel spray out of twenty laser ignition attempts. The volume flux is a fuel spray parameter that provides a measure of fuel flow per unit cross-sectional area of the spray at a particular location within the fuel cloud. This parameter was measured by placing a 0.125 -inch-diameter aperture within the fuel spray at each of the locations chosen for laser initiation. Fuel droplets passing through this aperture were collected within a vessel and the total volume of fuel collected over several minutes of operation was recorded. From this information, a value of volume flux was derived.

Figure 8 shows significant differences in the performance of the two laser ignition mechanisms as the flow of fuel in the region of the laser spark was decreased. Over-all, less laser energy was required of the dual pulse ignition mechanism compared to the single laser pulse approach for any of the fuel flow conditions addressed. Under richer fuel flow conditions, such as seen at the "sweet spot" location at a distance of $1 \mathrm{~cm}$ from the nozzle, the smallest difference between single and dual pulse energies required for consistent ignition was obtained $(\sim 50 \mathrm{~mJ})$. As the fuel became leaner, considerably more energy was required of the single igniting pulse compared to the dual pulse ignition results. At the "sweet spot" located at a longitudinal distance of $5 \mathrm{~cm}$ from the nozzle, no consistent ignition could be obtained utilizing the single pulse technique even at the maximum output pulse energy available from the laser source.

Experiment \#6 - Dual Pulse Energy for Consistent Ignition vs. Fuel Temperature. This experiment defined the dual pulse laser energy required to achieve $100 \%$ consistent ignition probability over a broad range of fuel temperatures. The DLP ignition method requires application of a first pulse to produce a light absorptive plasma within the fuel spray, then followed by application of a second laser pulse to sustain and energize the plasma resulting from the first pulse. For this experiment, the first laser pulse was provided by a Nd:YAG laser with a $12 \mathrm{~ns}$ duration output pulse and for the second laser pulse, a Cr:LiSAF laser with a $85 \mathrm{~ns}$ long output pulse was used. A $10-\mathrm{cm}$ focal length lens was used to focus the output of each laser onto the fuel cloud to be ignited. An ignition "sweet spot" located $1.0 \mathrm{~cm}$ for the nozzle face and $12.5 \mathrm{~mm}$ from the nozzle axis was chosen for the initiation location. The temperature of the fuel was reduced from an initial temperature of $85 \mathrm{deg} F$ to a final temperature of $-40 \mathrm{deg} F$, as measured directly at the fuel nozzle. A first pulse energy with a constant $120 \mathrm{~mJ}$ pulse energy was applied to the fuel, while the second pulse energy was increased from 0 to a maximum of $450 \mathrm{~mJ}$ as needed to obtain consistent ignition for any given fuel temperature. 
The results of this experiment are summarized in Figure 9. Here the percent increase in total laser energy from an initial value of $120 \mathrm{~mJ}$, as required to achieve consistent fuel ignition, is plotted versus fuel temperature. Total laser energy is the sum of first and second pulse energies. Each data point in the plot represents the results of twenty ignition trials. At temperatures above $+45 \mathrm{deg} F$, the first laser pulse energy, set at a constant $120 \mathrm{~mJ}$, was sufficiently energetic to ignite the fuel spray. Below a temperature of 45 deg F, additional laser energy, supplied by the second laser pulse, was required to obtain consistent ignition. Although the slope of the ignition curve below 45 $\operatorname{deg} F$ is fairly steep (about a $100 \%$ increase in laser energy per $14 \mathrm{deg} F$ decrease in fuel temperature), the curve shows that fuel ignition at temperatures as low as $-43 \mathrm{deg} F$ was achievable. One data point in the plot (the square) is ignition energy required using the Q-switched first laser pulse alone to ignite fuel at a temperature of $22.5 \mathrm{deg} F$. At 22.5 deg F, a 180-mJ (or 150\%) increase in Nd:YAG pulse energy was required for $100 \%$ probable ignition. At the next temperature setting examined ( 0 deg $F$ ), no consistent ignition of the fuel could be achieved with the single, Q-switched pulse alone up to the maximum output energy $(550 \mathrm{~mJ})$ of the Q-switched Nd:YAG.

Experiment \#7 - Dual Pulse Ignition Dependence On First Pulse Energy. Since the dual pulse ignition method requires two laser pulses, the following experiment defined the optimal partition of laser energy between first and second pulses. The first laser pulse was provided by a Nd:YAG laser with a $12 \mathrm{~ns}$ duration output pulse and for the second laser pulse, a Cr:LiSAF laser with a $85 \mathrm{~ns}$ duration output pulse was used. The temporal delay between application of the two laser pulse was set to $100 \mathrm{~ns}$. A 10$\mathrm{cm}$ FL lens was used to focus the output of each laser onto the fuel cloud to be ignited. An ignition "sweet spot" located $1.0 \mathrm{~cm}$ from the nozzle face and $12.5 \mathrm{~mm}$ from the nozzle axis was chosen for the initiation location. The fuel was stabilized at a reduced temperature of $-0.3 \mathrm{deg} F$, measured directly at the fuel nozzle. Ignition frequency data was collected over a range of second laser pulse energy with two settings of first pulse energy: 1) a pulse energy of $89 \mathrm{~mJ}$, which was $10 \mathrm{~mJ}$ above the minimum pulse energy required to break down air (79 $\mathrm{mJ})$ and 2) a substantially higher pulse energy of $123 \mathrm{~mJ}$. The results of this investigation are shown in Figure 10. Here, ignition frequency is plotted versus the energy contained in the second laser pulse, provided by the Cr:LiSAF laser. Each data point represents the result of twenty ignition trials.

With no laser energy provided by the Cr:LiSAF laser, the ignition frequency obtained for a pulse energy of $123 \mathrm{~mJ}$ using the Nd:YAG laser alone was higher than that obtained at a pulse energy of $89 \mathrm{~mJ}$, as expected. What was not expected was the resulting ignition frequency curves obtained as second pulse energy was increased from 
zero at each condition of $\mathrm{Nd}: \mathrm{YAG}$ first pulse energy. The ignition frequencies for the two conditions of first pulse energy increased at significantly different rates until $100 \%$ ignition reliability was achieved. Remarkably, the second pulse energy required to achieve $100 \%$ consistent ignition is virtually the same $(\sim 100 \mathrm{~mJ})$ for both cases of first pulse energies utilized. This implies that a substantial fraction of the totai fuel initiation energy deposited within the fuel cloud was contributed by the second laser pulse. Measurements made in a later experiment tend to support this conclusion, as will be demonstrated, although other effects, such as the prolonged deposition of energy from the second pulse, may also beneficially influence the outcome of the dual pulse ignition process.

\section{Experiment \#8 - Laser Energy Requirements for Dual and Single Laser Pulse}

Ignition. In this series of experiments, a somewhat harder to light region of the fuel cloud was chosen to initiate fuel combustion. The laser spark was positioned $2.5 \mathrm{~mm}$ from a "sweet spot" located at a distance of $1.0 \mathrm{~cm}$ from the nozzle and at a radial distance 10 mm away from the nozzle axis. The fuel flow volume flux at this position was measured to be about $.75 \mathrm{scc} / \mathrm{s}-\mathrm{cm}^{2}$. Data was collected in the manner described above, except that in this case both the dual pulse and conventional laser spark ignition methods were applied to ignite fuel cooled to a temperature of $-5 \mathrm{deg} C$. The results are shown in Figure 11, where the measured ignition frequency is plotted versus total laser energy applied. For the dual pulse case, the Nd:YAG pulse energy was kept constant at $80 \mathrm{~mJ}$, while the Cr:LiSAF second pulse energy was adjusted. Again, total energy is defined as the sum of energies contained in the first and second laser pulses for the dual pulse case.

Several significant features are apparent in the data: 1) the ignition frequency obtained by the dual pulse method increased considerably faster than for the single pulse initiation as laser energy was increased, 2) consistent ignition of the fuel could be achieved at a laser energy of about $200 \mathrm{~mJ}$ for the dual pulse case, whereas for the single pulse approach consistent ignition could not be obtained even at a pulse energy of 450 $\mathrm{mJ}, 3$ ) the low initiation probability at lower laser energies was approximately the same for the two ignition approaches, and 4) at a laser energy above $130 \mathrm{~mJ}$, the two ignition frequency curves diverge significantly. We suspect that many of the differences observed may be due to fundamental differences in the efficiency of light energy transfer to the igniting spark for the two fuel ignition methods, as predicted by theory. To quantify the energy transfer efficiency and to assist in the identification of the features present within the data of Figure 11, as well as other dual vs. single laser pulse ignition data, the following experiment was conducted to measure the fraction of laser energy transmitted through a laser breakdown spark. 
Experiment \#9-Efficiency of Laser Energy Utilization. This experiment measured the fraction of laser light transmitted through the focal volume of the lens used to focus fuel-igniting light to a power density sufficient to cause the breakdown of air molecules and the formation of an airborne plasma. The apparatus used for this measurement is shown in Figure 12. This experiment was performed to estimate the fraction of laser light energy utilized in the formation and heating of the laser-induced spark by monitoring the fraction of unabsorbed laser energy transmitted through the laser spark region. The hypothesis applied here is that the deficit of laser energy measured in the light transmission through the laser spark volume is proportional to the light energy absorbed by the plasma, which is subsequently converted to fuel igniting thermal energy.

Measurements were performed for both the SLP and DLP methods over a range of laser energy. Uncoated beam splitters were placed before and after the breakdown region to permit a small fraction (several percent) of the incident and transmitted light to be diverted for energy measurement. In order to eliminate detector response variations, a single pyrometric energy monitor was used to measure the laser energy before and after the spark-breakdown region. This was done by physically moving the detector from point $A$ to point $B$ in the drawing. Total incident energy was measured by placing the detector at position C. A constant first pulse energy of $80 \mathrm{~mJ}$ was used for the dual pulse studies.

The result of the measurements are shown in Figure 13, where the fraction of light energy transmitted through the laser spark region is plotted vs. laser energy input to the plasma formation region. The $100 \%$ transmission point corresponds to a pulse energy of $53 \mathrm{~mJ}$, which was just below the laser energy threshold for the formation of a visible laser spark. For the DLP transmission measurements, both first and second pulse energies were monitored as the total laser energy. The results show that at lower laser energies, the fraction of transmitted laser energy was approximately the same for both single and dual pulse beams. At higher laser energies, the fraction of transmitted laser light was considerably less for the DLP technique relative to that obtained by the single pulse method for the same total laser energy. For example, at a total laser energy of $250 \mathrm{~mJ}$ about $52 \%$ of the incident light energy was transmitted through the laser spark region when this energy was contained within a single $12 \mathrm{~ns}$ laser pulse. This is in contrast to $23 \%$ for the dual pulse case at the same incident total laser energy of $250 \mathrm{~mJ}$.

This result is expected since for the single pulse case no appreciable laser light can be absorbed by the transparent air until a light absorptive plasma is produced. Typically the laser spark is not created until sufficient power densities of the order of $10^{11 \mathrm{~W} / \mathrm{cm}^{2}}$ are provided by the laser pulse. Such power densities are typically obtained 
near the peak intensity of the laser pulse, leaving the earlier-in-time, lower-intensity portion of the laser's temporal pulse to pass unabsorbed through the transparent air. For the dual pulse case, the laser-induced plasma is produced at a pulse energy near the threshold for laser spark formation and relies upon the efficient absorption of the laser energy contained within the second laser pulse, which typically is applied $100 \mathrm{~ns}$ after the formation of a light absorbing plasma by the first laser pulse.

Although what had been measured in these experiments was the transmission of laser light through the plasma region, the inverse property of light absorption within the plasma is implied. If one assumes that the deficit of transmitted light through the spark plasma is proportional to the light absorption within the plasma, then comparisons of absorbed laser energy for first and second laser pulses can be made, neglecting light scattering. Using this assumption and the prior comparison cited, the quantity of absorbed light for the dual pulse case was $20 \%$ greater than that provided by application of a single laser pulse alone with the same energy content. The greater laser energy coupling efficiency of the DLP approach can be demonstrated further in the following manner. In order to deposit $50 \%$ of the laser energy in the plasma by the application of a single laser pulse, about $100 \mathrm{~mJ}$ more laser pulse energy is required compared to that required via application of a dual laser pulse approach, as shown in Figure 13. The measured improvements in laser energy utilization efficiency is expected in light of the observed enhancement in fuel ignition performance provided by the dual laser pulse fuel ignition method.

To further illustrate the significance of the two curves of Figure 13, an analysis of the fraction of second laser pulse energy transmitted through the plasma region was made. The plot of Figure 14 shows the fraction of transmitted second laser pulse energy as a function of incident second pulse energy upon the spark region. The laser spark was initially produced by application of the first pulse, provided by the Nd:YAG laser operated at a pulse energy of $80 \mathrm{~mJ}$. The curve of Figure 14 shows a very rapid decrease in transmitted second pulse as second pulse energy was increased, until a second pulse energy of about $45 \mathrm{~mJ}$ was applied to the laser spark. At this laser energy, the transmission of second laser pulse light through the laser spark plasma assumes a nearly constant value of between 10 and $20 \%$. Apparently at this second pulse energy, the spark plasma had nearly completely ionized the local air molecules, obtaining a virtual maximum in its optical density. As laser energy is further increased, little change in plasma transmission (or absorption) was obtained--the plasma saturated in its light absorption property. 
This saturation effect is observed in several of the previous data plots. For instance, the dual pulse ignition curve of Figure 11 shows a prominent knee in the slope of the ignition frequency curve at a total laser energy of about $125 \mathrm{~mJ}$ or, equivalently, at a second pulse energy of $45 \mathrm{~mJ}$. This corresponds well with the observed saturation of the second laser pulse transmission (or absorption), which occurred at an energy of $45 \mathrm{~mJ}$ as seen in Figure 14.

Also, the data of Figure 10 shows that consistent dual-pulse fuel ignition was not obtained until a minimum second pulse energy of about $100 \mathrm{~mJ}$ was applied to the laser spark, regardless of the energy contained within the first laser pulse that created the airborne plasma. This result was not expected, and may relate to a situation in which no appreciable laser energy was deposited by the second laser pulse until the resulting plasma became dense enough to efficiently absorb the second pulse of laser light. The experimental results shown in Figure 14 indicate such an effect, where plasma absorption does not become significant until a second pulse energy of about $45 \mathrm{~mJ}$ is applied to the plasma. At this energy, the transmission of the plasma had decreased to a value of about $15 \%$. As noted in the lower curve of Figure 10, the ignition frequency began to rise rapidly at approximately this value of second laser pulse energy. With a further increase in second pulse energy, consistent ignition was obtained at a second laser pulse energy of about $100 \mathrm{~mJ}$ for both conditions of first laser pulse energy. Using the plasma transmission curves of Figure 13, the fraction of absorbed light for the 89 and $123 \mathrm{~mJ}$ Nd:YAG laser, first pulse laser energies are about 15 and 25\%, respectively, corresponding to absorbed light energies of about 13 and $31 \mathrm{~mJ}$. At the second pulse energy of $100 \mathrm{~mJ}$, an effective light energy absorption of about $85 \%$ is obtained, providing $85 \mathrm{~mJ}$ to the plasma. This absorbed second laser pulse energy is much larger than the absorbed energy contribution from the first pulse at either of the input energies of 89 or $123 \mathrm{~mJ}$, which is consistent with the observed ignition dependence upon second pulse energy.

\section{Allied Signal Demonstration}

Discussions of our ignition technology led to a collaborative effort with Allied Signal (Phoenix) to investigate the relative benefits of the dual-pulse ignition technology to ignite turbine engines under actual turbine conditions of fuel/air flow and combustion chamber pressure. Allied Signal is a leading manufacturer of intermediate-sized turbine engines used for auxiliary power generation in aircraft and has an active laser-induced ignition $R \& D$ program of its own, with the goal of developing a laser igniter, primary for use in auxiliary power turbines. In this collaborative effort, we were invited to evaluate the dual-pulse technology on Allied Signal's turbine combustor test rig at their 
Morristown, NJ Technology Center. Direct comparison of the performance of the DLP igniter was made to that obtained by Allied Signal using their SLP approach.

The combustor test rig at Allied Signal is a mock-up of a turbine combustor that operates at the actual air and fuel flow rates present within an auxiliary power turbine engine. It consists basically of the combustion chamber, a fuel injector and pumps to draw air and fuel into the combustor volume at appropriate rates and pressures. A laser window at the upper surface of the combustion chamber allows laser light to be introduced into the combustor rig. The tubular window assembly included a 1-indiameter focusing lens as well as the laser window.

The dual-pulse laser apparatus used in this study has the same configuration shown in Figure 1, except that a 2.5X telescope was used at the output of the laser system to collimate the laser output in transport to the combustor. The higher output Nd:YAG laser used in previous dual-pulse experiments at LANL was replaced by a physically smaller Nd:YAG laser to permit installation of the Nd:YAG and the Cr:LiSAF lasers on a small 1.5x 2-ft breadboard. The maximum pulse energy for the multiple transverse mode output of this Nd:YAG laser was $150 \mathrm{~mJ}$. During these tests, the dual pulse laser was operated at several different values of Nd:YAG and Cr:LiSAF output pulse energies, with the combined laser energies being adjusted in the range of 78 to $193 \mathrm{~mJ}$. The temporal delay between the first and second laser pulses was set to 100ns. The dual-pulse laser breadboard was set up adjacent to the test rig and its output propagated horizontally a distance of about $3 \mathrm{~m}$ to a turning prism, which directed the laser light downward into the combustor window. A 12-cm FL lens was used for the dual-pulse investigation, whereas a $15-\mathrm{cm}$ FL lens was used for the Allied Signal laser. This lens was slightly longer than the $10-\mathrm{cm}$ FL lens used for most of our previous dual-pulse studies but was needed to project a laser spark to the appropriate location within the combustor. The initiation site chosen for the laser spark was near that used during the Allied Signal tests. Due to limited adjustment in the positioning of the focusing lens, the dual-pulse initiation site was slightly higher from the combustor axis than the desired location by about 0.1 inches.

For the experimental program, the combustor was operated over the same range of air and fuel flows used by Allied Signal in their laser-induced ignition program. A major difference between the mode of operation of our and Allied Signal's lasers was the pulse repetition rate. Allied Signal's ignition tests were performed at a $20 \mathrm{~Hz}$ pulse rate, while our DPL system was operated at a $1 \mathrm{~Hz}$ rate. This led to some complications due to the manner in which combustor control and data collection systems operate. The Allied Signal combustor was designed to operate over a maximum time interval of ten seconds. For the dual-pulse igniter, which operates at $1 \mathrm{~Hz}$, only ten shots of the laser could be 
applied during this operating time. This limited our ability to evaluate the igniter performance in hard to light regions of the fuel/air flow regime. In addition, the windows and focusing lens incorporated into the combustor's light feed-through system were antireflection coated for $1064 \mathrm{~nm}$ light, which was partially reflective to the $850 \mathrm{~nm}$ light produced by the Cr:LiSAF laser of our DPL system. The transmission of these optical components to $850 \mathrm{~nm}$ light (52\%) was measured and an adjustment was made to the 850 $\mathrm{nm}$ energy utilized during the ignition tests to correct for these reflective losses. Some minor vignetting was also observed in the dual-pulse light caused by the larger beam diameter of the dual-pulse laser's output.

The results of this investigation are summarized in Figures 15-18. Figure 15 shows the results of Allied Signal's laser igniter tests using a single Q-switched pulse with an energy of $125 \mathrm{~mJ}$ and a pulse width of about $9 \mathrm{~ns}$. What is shown is an ignition map obtained over a range of air flows on the horizontal axis and a range of fuel flows on the vertical axis. The regions in the plot labeled by a letter refer to the number of laser shots required to achieve fuel ignition at that particular value of air and fuel flow. A key, located in the upper corner of each plot, relates the labeling letter to the number of laser shots required for ignition. As seen from the plot, Allied Signal was capable of achieving combustor ignition within several shots of their laser over most of the air and fuel flow regime, except for the region in the lower right hand corner of the plot, which represents lean fuel and high air-flow-rate conditions. In this region, the SLP igniter required as many as 50 laser shots or more to ignite the combustor.

Figures 16 and 17 show the results obtained by our DPL igniter operated at total laser energies of 112 and $135 \mathrm{~mJ} /$ laser shot. For these cases, the Nd:YAG laser was operated at a pulse energy of $78 \mathrm{~mJ}$, while the Cr:LiSAF laser was operated at 34 and 57 $\mathrm{mJ}$, respectively. Ignition was obtained within 10 shots over nearly the entire fuel and air flow range. The exception was the hard to light region of lean fuel and high air flow rate, where no ignition was induced within the ten laser shot limit. In general, ignition could be achieved using the dual-pulse igniter with considerably fewer laser shots ( factor of two to three times less shots) compared to the Allied Signal results utilizing approximately the same laser energy per laser shot. This result has considerable impact on the design of an igniter laser system, since a factor of two lower time-averaged power is required to achieve consistent ignition, thus reducing the weight and volume of the laser and laser power supply. At somewhat higher DPL energy (193 mJ), the ignition envelope was considerably extended to higher regions of air flow, and provided ignition within ten laser shots over the full range of fuel at air flows up to $0.163 \mathrm{lb} / \mathrm{s}$, as shown in Figure 18. 


\section{Ignition Laser Hardware Design Effort}

Although we have developed and demonstrated an efficient and reliable laser ignition method, all lasers utilized were large and fragile laboratory-scale systems. To further the development and application of the DLP ignition technology will require the realization of compact and durable dual pulse laser hardware. This is particularly true for the deployment of this technology to aircraft turbine engines. The aircraft turbine engine environment in which a laser igniter must operate is extremely harsh. Such an igniter will experience substantial G-forces, severe vibrations, extremes in temperature and exposure to grime, moisture and corrosive substances such as fuel and combustion resides. The laser igniter must also be able to withstand substantial duty factors in their operation. Besides the survival issues, the laser igniter must be compact, energy efficient, low maintenance and cost effective relative to competing igniter technologies, particularly in relation to commercial applications. To address these issues, a DPL design concept has been conceived and is described below.

One DPL igniter concept that shows great potential is shown in Figure 19. This laser system basically consists of four components: 1) a small, solid-state fuel igniting laser that resides in the aircraft engine, 2) a second solid-state laser located remotely from the igniter laser and engine that provides excitation energy to the first laser in the form of an optical pulse, 3) an optical fiber linking the two lasers that transports the igniter laser excitation light, and 4) a fiber multiplexer to switch the excitation light from one exciter laser to many individual igniter lasers. The light output of the exciter laser is set to a wavelength capable of energizing the igniter laser. A single laser pulse from the exciter laser induces the generation of the required dual pulse format at the output of the igniter laser by a sequential process of passive Q-switching and gain switching. The primary advantages of this approach are: 1) compactness, durability and cost-effectiveness of the fuel-igniting laser are substantially improved by eliminating any of the usual sources of laser excitation such as fragile flashlamps or costly diode lasers at the igniter laser, 2) the igniter laser construction becomes virtually monolithic with no moving parts or electrical input of any kind required, further improving durability and reducing maintenance, 3) a single excitation laser can be used to energize many individual igniter lasers, substantially reducing cost, and 4) routine maintenance is confined largely to the single excitation laser that resides in the controlled environment of the aircraft's cabin.

The excitation laser is a solid-state laser device capable of producing a shortduration light pulse at a wavelength of $808 \mathrm{~nm}$ as required for the excitation of the igniter laser. The primary candidate for this laser is a slow, Q-Switched, Cr:LiSAF laser, tuned to operate at the $808 \mathrm{~nm}$ wavelength. Slow Q-switching of the laser would achieved by 
the appropriate modulation of the laser's active Q-Switch. In order to appropriately excite the igniter laser, approximately $150 \mathrm{~mJ}$ is required within a $200 \mathrm{~ns}$ duration laser pulse. Although this material can be energized by laser diode pumping, a more practical pumping mechanism would be provided by flashlamps. A major drawback of flashlamps is a relatively short lifetime, requiring replacement typically after 30 million laser shots, although their cost-effectiveness outweighs other potential energizing sources such as laser diodes.

The diagram of Figure 19 shows the utilization of a fiber multiplexer to distribute the light output of a single excitation laser to many individual igniter lasers, located at either different engines of the vehicle or at different points within the same engine. Although a single, remote excitation laser can be utilized with each igniter laser, use of a fiber switching system substantially reduces cost, since only one excitation laser is required. One possible configuration for a fiber switching system is the electro-optically controlled fiber switch shown in Figure 20. This prototype device was developed and evaluated with support from the DoD program office. This device can operate at switching speeds up to $1 \mathrm{kHz}$ and at light power densities as high as $300 \mathrm{MW} / \mathrm{cm}^{2}$. The optical fiber switch consists of alternating pairs of polarizers and Pockel's cells, which are capable of directing light to optical fibers located at the escape ports of each polarizer in the chain. Light switching is accomplished by applying a voltage to the Pockel's cell with sufficient amplitude to rotate the original linear polarization by 90 degrees, permitting rejection of the light at the next polarizer in the laser light's propagation. LiNO ${ }_{3}$ Pockel's cells were used for the prototype device. A major benefit of this type of laser light switching system is that it has no moving parts to cause misalignment of the light injection into an optical fiber and the potential fiber damage that can result. A variation of this device, which may work well at lower switching rates while reducing cost, replaces the fast Pockel's cell utilized previously with rotatable half-wave plates.

The transport of light between the excitation laser and the igniter laser is performed by a 500-micron core diameter, multiple-mode optical fiber. A light injection and typical transport efficiency of about $85 \%$ is expected for fiber lengths of $10 \mathrm{~m}$ or less. The light power density within the fiber is less than $300 \mathrm{MW} / \mathrm{cm}^{2}$, which is well below the threshold for optical damage within the fiber of about $3 \mathrm{GW} / \mathrm{cm}^{2}$.

The igniter laser consists of a solid-state lasing crystal, a passive, solid-state Qswitch, an optically resonant cavity in which the reflective elements are composed of highly reflective coatings deposited upon the laser crystal and Q-switching material, and a focusing lens for the laser output, as shown in Figure 21. These components are rigidly 
mounted within the laser housing. Several types of solid-state lasing material can be used for the lasing medium, although Cr:Nd:GSGG is preferred due to its high gain and mechanical strength. This laser is excited by a 200 -ns duration, $808-n m$ wavelength light pulse output from the remote excitation laser. A graded refractive index lens is used to collect excitation light from the transport fiber and to focus this light into one end of the lasing crystal. The mechanism for producing a dual-pulse output from this laser is described below.

The high peak power of the excitation light rapidly establishes a photon flux within the laser cavity capable of bleaching the saturable Q-switching material (Cr:YAG). A several nanosecond-duration Q-switched pulse with a pulse energy of about $30 \mathrm{~mJ}$ is produced at the output of the igniter laser early within the duration of the excitation pulse. When focused by the output lens, a sufficient power density is achieved at the focal plane to produce a laser spark. Following the output of the Q-switched pulse, the excitation light continues pumping the lasing material and prevents the recovery of the Q-switch to its lower transmittance state, and the laser continues to output light, in a gain-switched manner, over the remaining duration of the excitation light pulse within the laser crystal. The result is the output of a long-duration laser pulse with a temporal length of about $85 \mathrm{~ns}$ (FWHM) and a pulse energy of about $60 \mathrm{~mJ}$. The separation in time between the appearance of the two pulses measured peak to peak is about $100 \mathrm{~ns}$. The resulting pulse format is precisely what would be required of the dual-pulse igniter based upon previous evaluations.

\section{Development of a Laser Ablation Ignition Method}

Before the cold fuel ignition studies were begun, a short series of experiments were conducted to determine whether fuel ignition could be achieved using longduration, low-peak-power laser light interacting with a light absorptive material surface to produce a hot, fuel-initiating vaporization plume.

Initial experiments employed long-duration laser light pulses (120 microseconds FWHM) obtained from a solid-state Er:YAG laser operating at a wavelength of 2.94 microns. The apparatus used in this demonstration is shown in Figure 22. The Er:YAG laser was operated in the free-running mode and provided a maximum output pulse energy of $400 \mathrm{~mJ}$. The laser was typically operated at a pulse repetition rate of $1 \mathrm{~Hz}$. A multiple transverse spatial mode was used for this demonstration. Emitted laser light was directed to a 0.5 -meter focal length lens, which was used to focus the laser light to a spot size diameter of about $1.0 \mathrm{~mm}$ at the focal plane of this lens. A fused silica lens was used for efficient transmission of this wavelength of light. 
To generate a hot vaporization plume, a target composed of a material that efficiently absorbs the 2.94-micron laser light was placed at the focal point of the focusing lens. The surface of the target was oriented to provide near normal incidence of the focused laser light relative to the plane of the target surface. Several target materials were tested for this application. The best performance with respect to the degree of material vaporized and the spatial extent of the vaporization plume generated was obtained using targets of graphite (carbon) or a metal alloy of iron and cerium. At a laser pulse energy of $400 \mathrm{~mJ}$, a white-hot, incandescent plume of vaporized material is produced that extended 2 to $4 \mathrm{~cm}$ from the target surface. The target material surface was oriented so that the focused laser light would be incident orthogonally. This vapor plume emanated from the target surface at a near 90 degree angle to the plane of the target surface and propagated in the direction toward the light source.

The ability of the laser-induced vaporized cloud to initiate fuel combustion was evaluated utilizing Jet A fuel aerosols, produced by a duplex, pressurized fuel injector of a type commonly used in commercial turbo-jet aircraft. The injector was operated at a differential pressure of 20 psi with ambient temperature fuel. To ignite this fuel, the target material was located adjacent to the fuel injector nozzle in a manner that permitted the laser-induced vaporization plume to intercept the fuel cloud as shown in Figure 22. Typically, the ablative target was placed $1.0 \mathrm{~cm}$ away from the face of the nozzle and 1.0 $\mathrm{cm}$ from the center of the nozzle in the lateral direction away from the laser. At this position, the laser target was kept just outside the periphery of the fuel cone to prevent direct contact of the fuel spray and target surface. The target was positioned so that the laser light, focused upon the target using the 50-cm FL lens, had to pass through the fuel cloud. The geometry of the target material was $1-\mathrm{cm}$ diameter, $1-\mathrm{cm}$ thick cylinder of graphite.

Ignition tests were conducted over a range of laser pulse energy. Limited time allotted for these experiments restricted ignition tests to the use of the 50 -cm focusing lens only. The vaporization plume from the target surface projected several centimeters into the fuel cloud and formed a nearly normal incidence to the nozzle spray axis. The spatially extensive vaporization plume, which intercepted a substantial fraction of the fuel cloud cross-section, resulted in $100 \%$ consistent fuel ignition at pulse energy settings of $150 \mathrm{~mJ}$ and greater. Typically, twenty laser-induced ignition attempts were performed at each energy setting. After each twenty-laser-shot ignition test sequence, the incident laser light would be repositioned at a new portion of the material surface. This was done to prevent severe erosion of the material. The laser light after each laser shot was observed to burn a cylindrical hole into the target surface about 2-mm deep and 1-mm in 
diameter. If the laser were kept focused upon one location of the material surface, a long narrow hole would be bored into the material. This would cause the width of the vaporization plume emanating from this hole to become narrower in successive laser shots (due to channeling of the hot vapors by the hole wall), and consequently cause degradation in igniter performance. To prevent such modifications to the vaporization plume caused by this mechanism, the focused laser light would be repositioned to a new area of the target surface following each twenty-laser-shot test sequence.

The high reliability of this ignition approach, obtained with a relatively simple low-pulse-energy, low-peak-power, solid-state laser, is very attractive for fuel ignition, although the erosion of target material limits igniter lifetime and adaptation to most fuel ignition applications. Specialized fuel ignition applications requiring short operating time and small laser size, weight, power and cost are best suited for this ignition approach. The operating lifetime of this laser-induced ignition could be substantially improved by optimizing target surface area and by illuminating successive regions with the laser light so as to ablate the material in an even manner. An eccentrically rotating target, relative to a fixed laser light focal point, is one means of prolonging ablative target life. A patent is pending on this ignition method.

\section{Summary}

The initial phase of this project consisted of a CRADA with B. F. Goodrich Aerospace and Laser Fare, Inc. in which we investigated two technology paths to advanced fuel-ignition concepts based on both laser-assisted and microwave-based ignition approaches. The laser-ignition portion of the CRADA effort resulted in the development and evaluation of two novel, laser-induced, jet-fuel-ignition concepts. These concepts utilize multiple laser wavelengths and pulse formats to enhance fuel ignition performance. The first of these novel concepts, the dual-laser-pulse (DLP) ignition method, utilizes the laser light pumping of a previously established laser spark to actively heat the fuel medium. A second ignition concept, called the laser preheat ignition method, utilizes the inherent light-absorptive property of the fuel at a 3-micron wavelength to vaporize aerosol fuel droplets prior to gas-phase ignition via a laserinduced spark. As part of this phase, we investigated the ignition of jet fuel sprays under ambient conditions. We compared ignition performance with a more conventional single-laser-spark (SLP) and found significant improvements in fuel ignition performance including lower laser energy requirements for consistent fuel ignition and lesser ignition sensitivity to fuel composition variations. A laser ablation method, capable of removing fuel residues from optical surfaces, was developed and evaluated experimentally. 
The experimental efforts conducted in the post-CRADA phase of this project were designed to advance our DLP technology to a variety of potential DoD application areas. Experiments were conducted demonstrating the reliability and energy efficiency of the DLP fuel ignition concept to 1) ignite jet fuel under ultracold conditions and 2) ignite jet fuel under realistic turbine engine flow conditions. Furthermore, laser hardware design concepts were developed that address many of the laser criteria essential to the further evaluation and deployment of the DLP technology to field applications including aircraft and ground-based vehicles and equipment. Some of the laser hardware concepts developed have been utilized in weapons development areas outside of the fuel ignition regime. Seven patents have been either issued or are pending that resulted from the technology developments in this project.

Our work has resulted in the establishment of many contacts in the government, military, and industrial sectors with vested interests in laser ignition. Some of these contacts have led to collaborative programs with Allied Signal Corp. and CFD Research Corp., as well as externally supported research for the U.S. Army. Several other collaborative efforts are currently being negotiated with the DoD in the area of laser stabilization of scramjet engines and ignition of rocket engines, and with private industry in the areas of power turbine stabilization and the ignition of nonhypergolic thruster rockets. Spin-off areas adapting laser ignition and laser igniter hardware concepts are currently being pursued within the DOE in the area of weapons safeguards and quality assurance. 


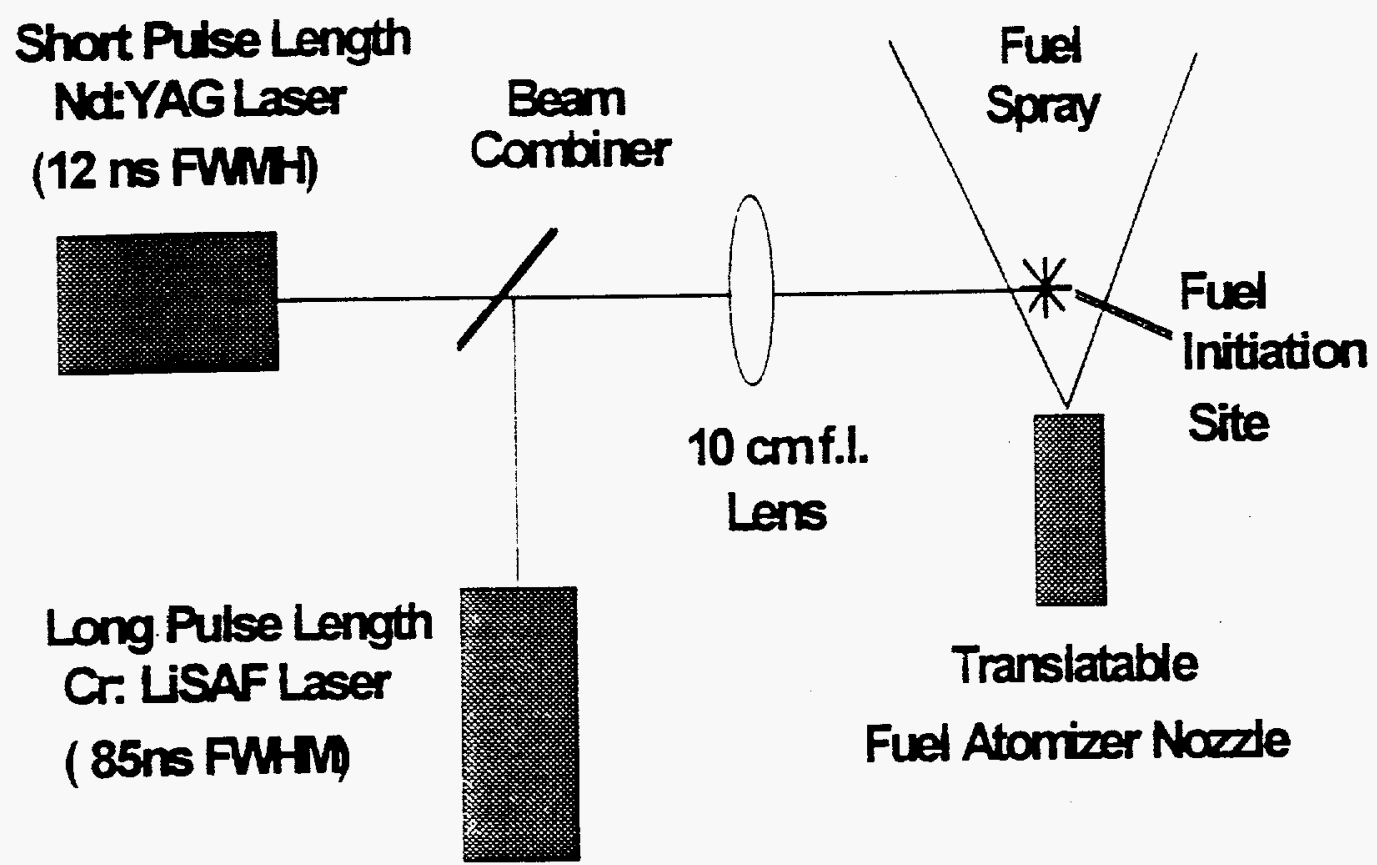

Figure1. Dual-pulse laser apparatus. 
Volume Flux Distributions at Various Distances from Nozzle

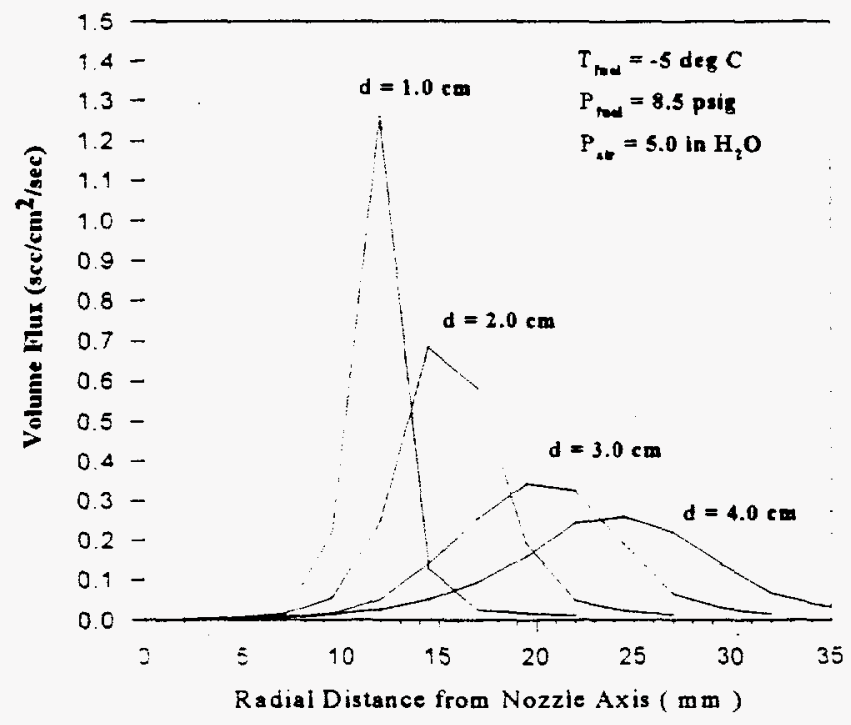

Figure 2a: Fuel droplet volume flux distributions.

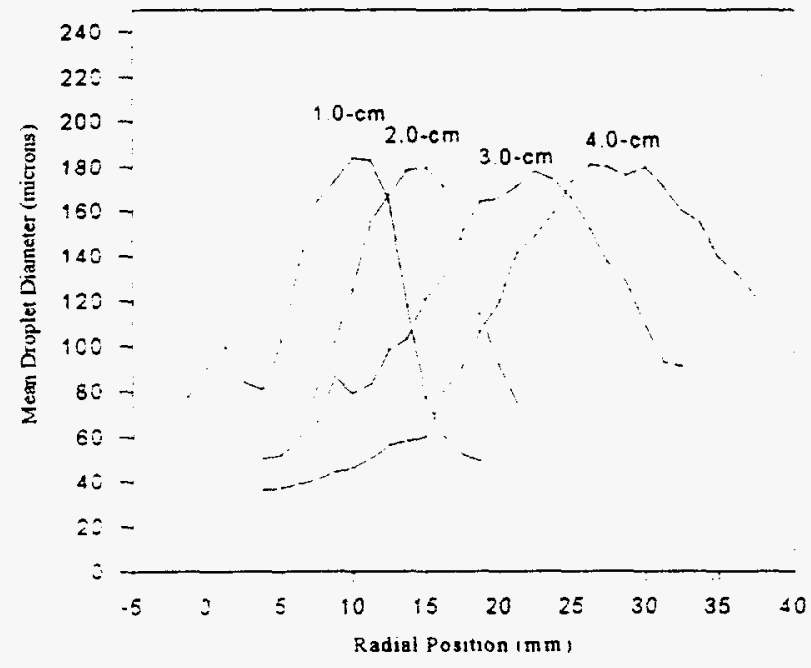

Figure 2b: Fuel droplet mean diameter (SMD) distributions. 


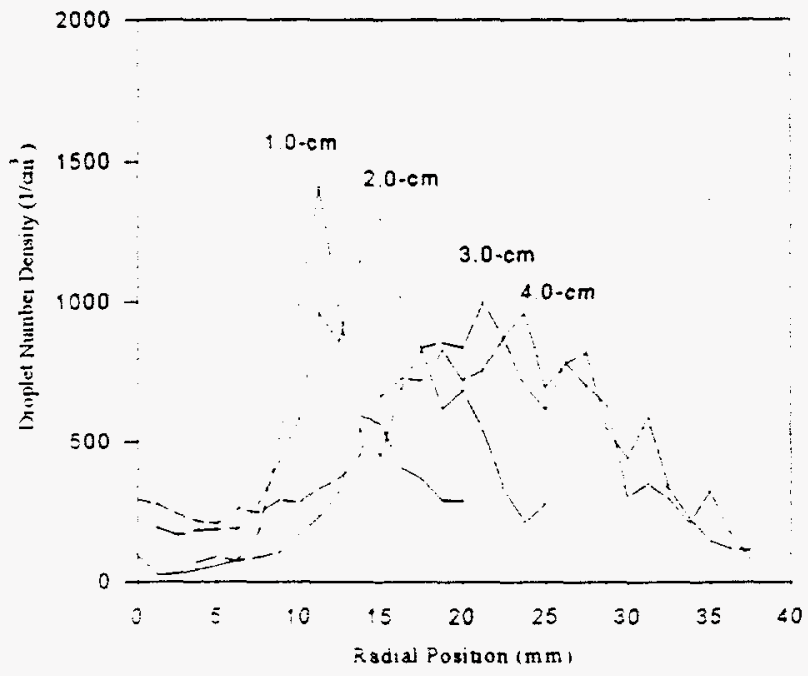

Figure 2c: Fuel droplet number density distributions.

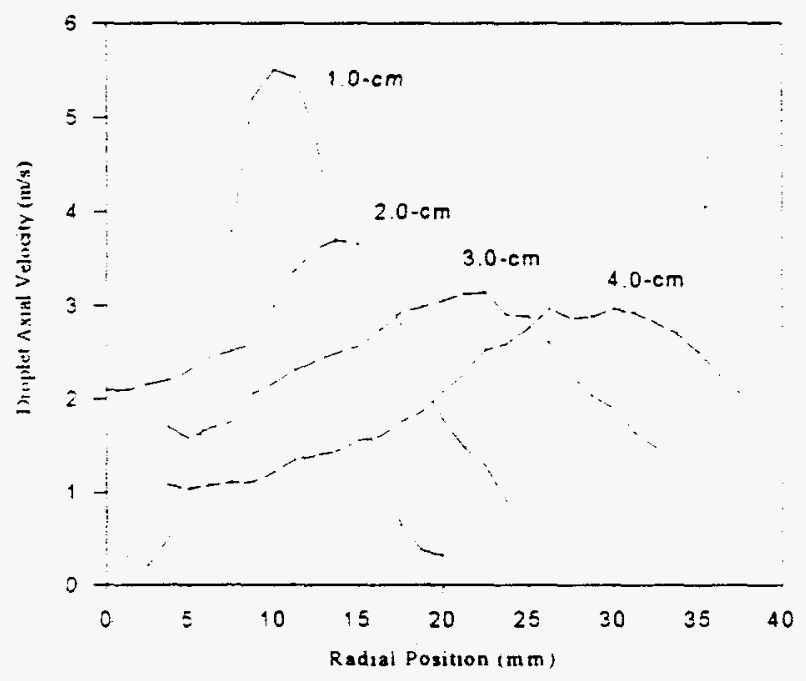

Figure 2d: Fuel droplet axial velocity distributions. 


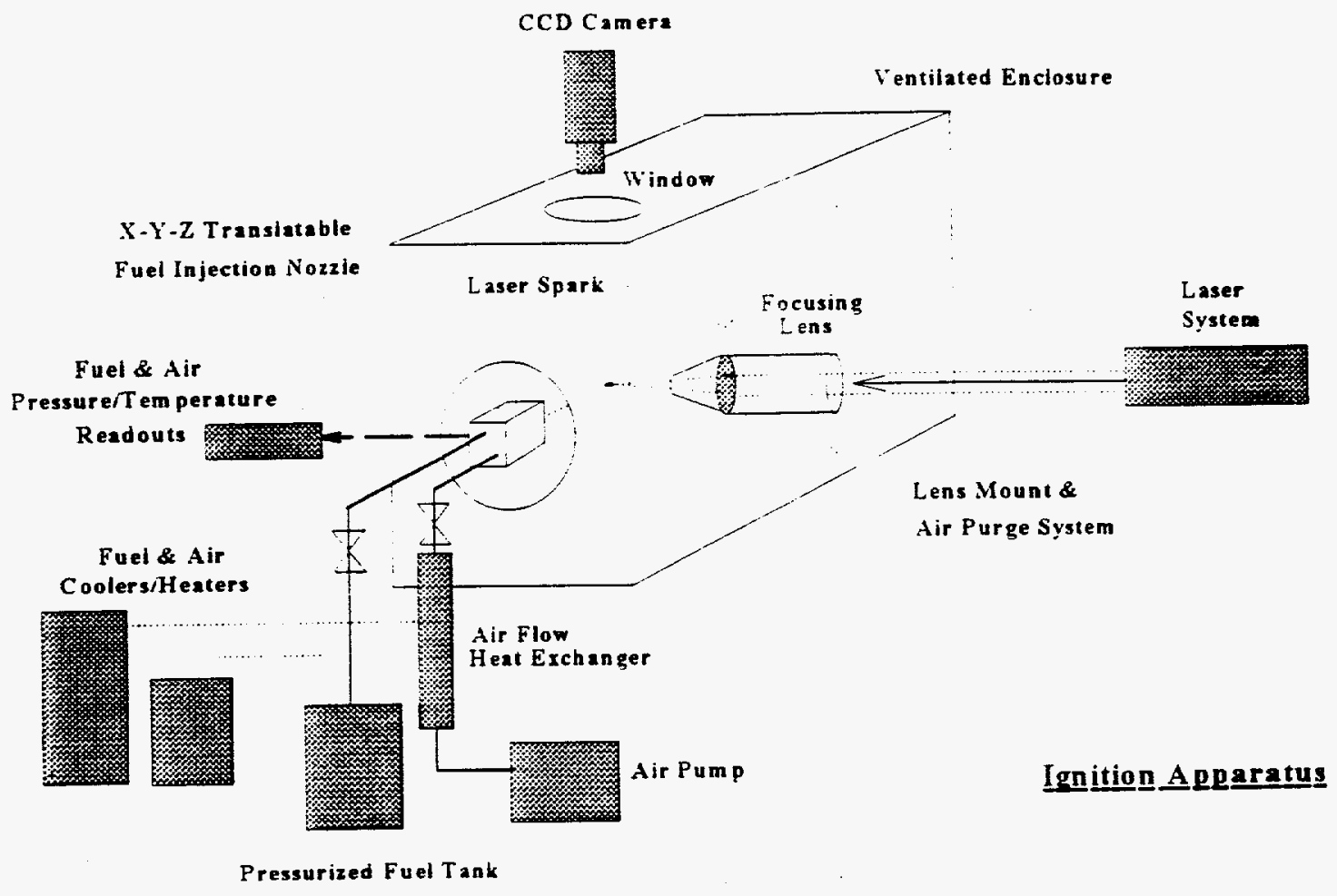

Figure3: Fuel ignition apparatus. 


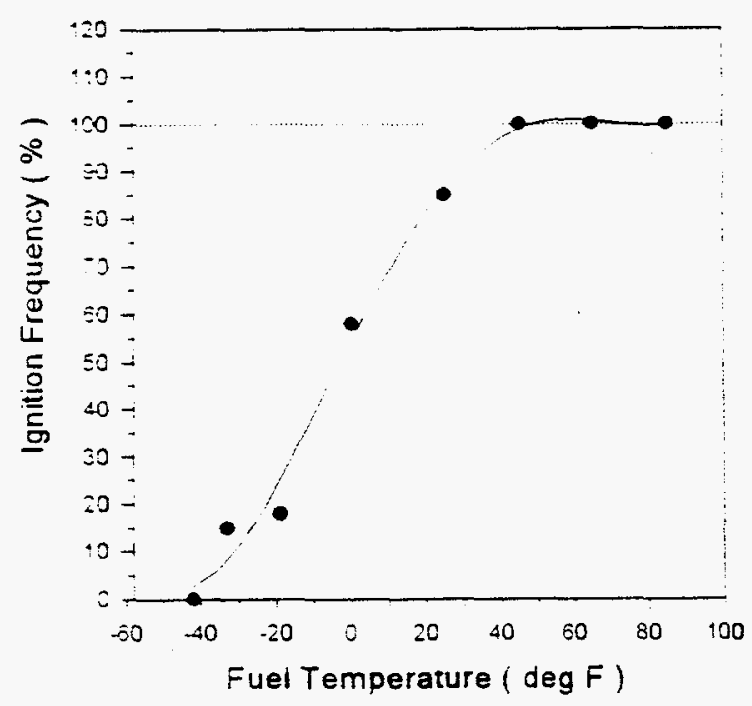

Figure 4. Temperature dependence of fuel spray ignition at a constant laser pulse energy of $120 \mathrm{~mJ}$.

Ignition Performance vs Distance from Nozzle

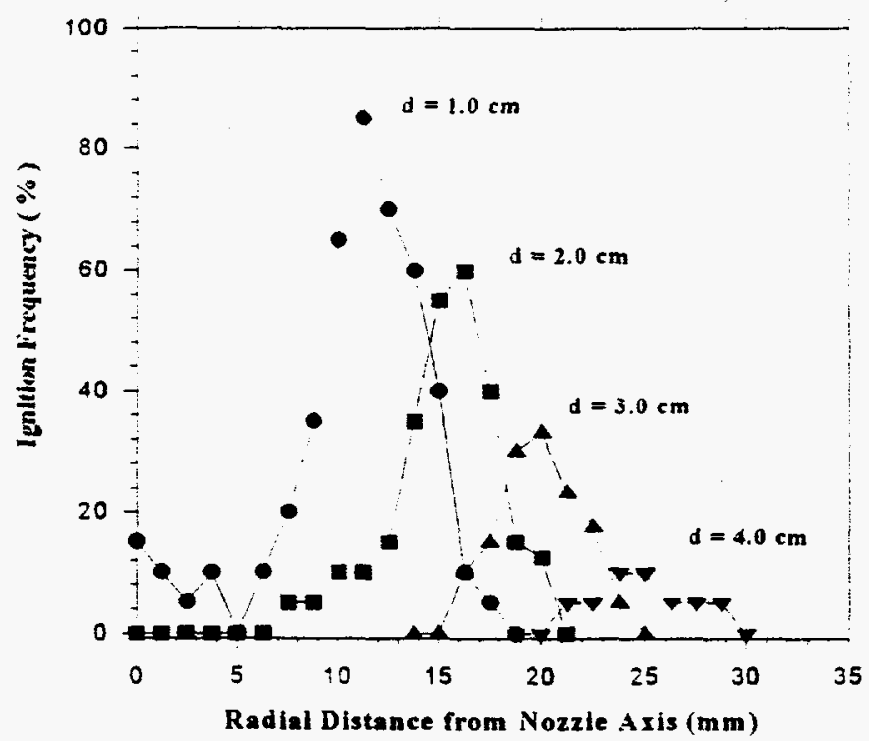

Figure 5: Radial mapping of ignition performance at various distances from the injector. 


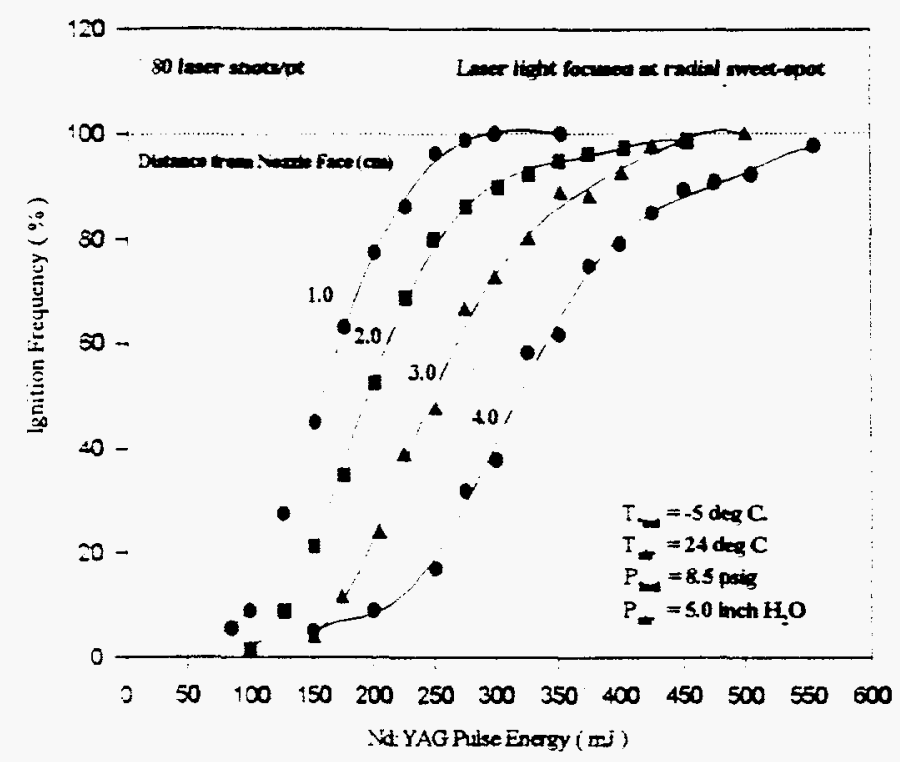

Figure 6. Ignition frequency vs. pulse energy for the single laser pulse(SLP) ignition approach.

Ign. Probabllity vs Temporal Delay

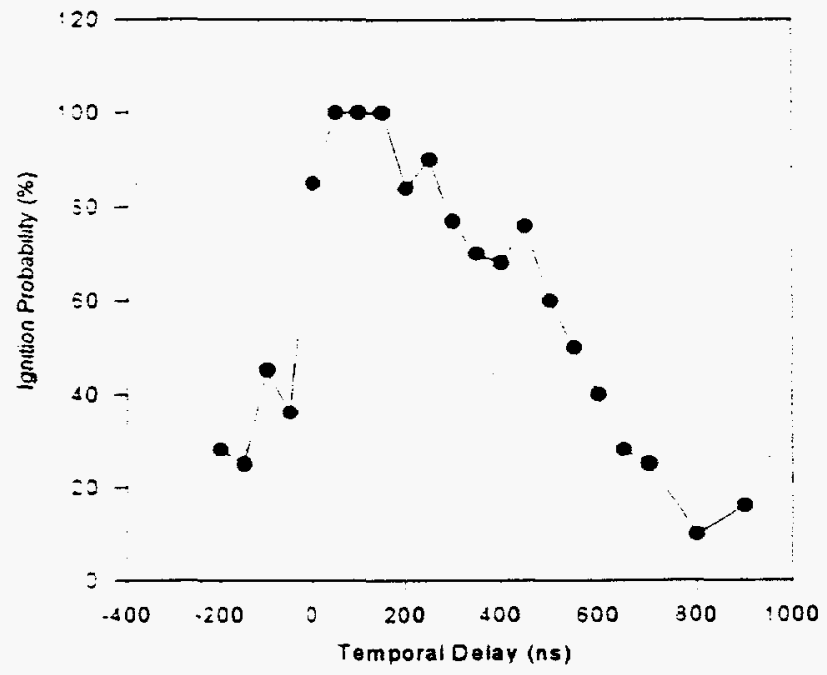

Figure 7: Ignition frequency vs. temporal delay for the dual pulse ignition format. 


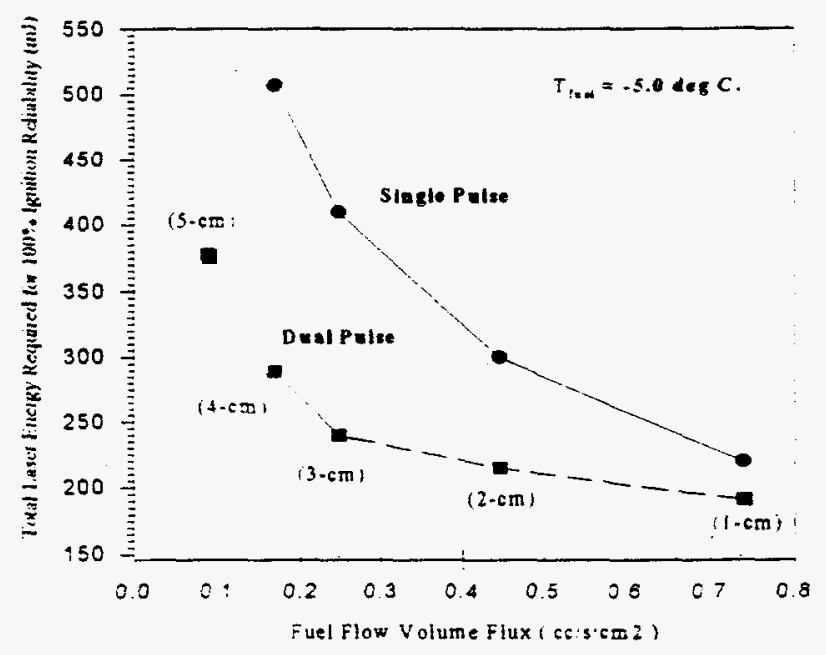

Figure 8. Dual laser puise vs. single pulse ignition--minimum laser energy required for consistent spray ignition vs. a fuel flow parameter.

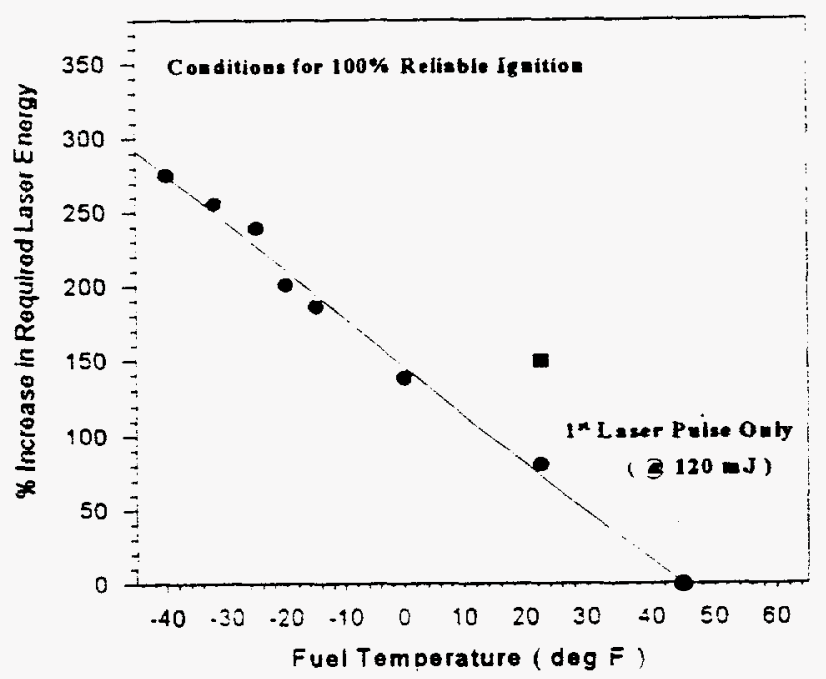

Figure 9: Required total laser energy for consistent ignition vs. fuel temperature. 


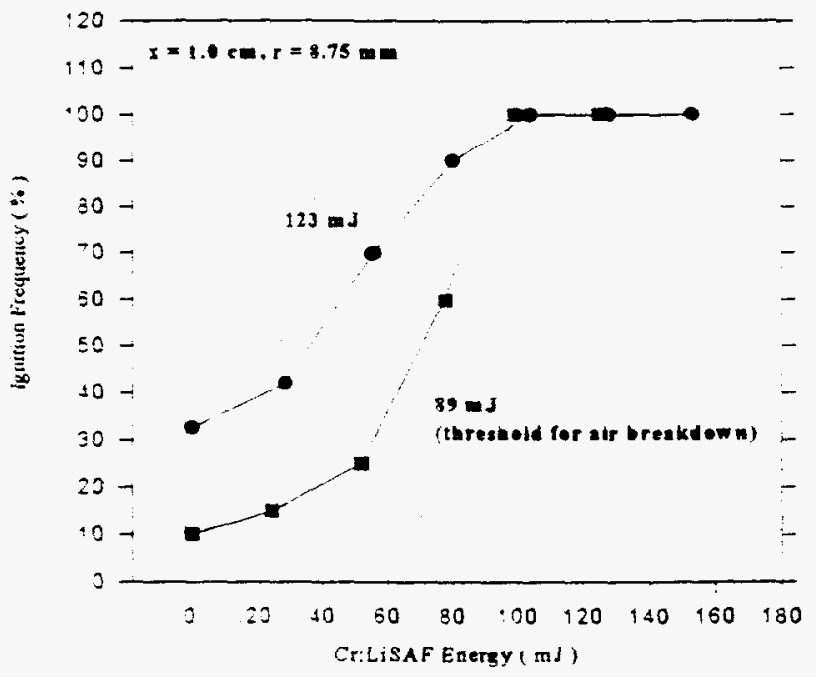

Figure 10. Ignition frequency vs. second laser pulse energy for two settings of first laser pulse energy.

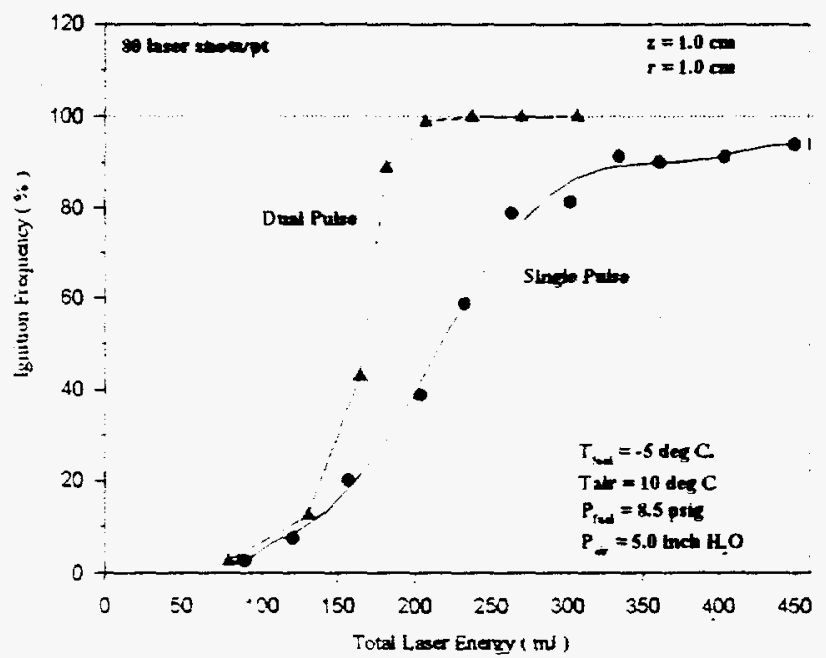

Figure 11. Dual pulse ignition provides reliable ignition with less total laser energy. 
$10 \mathrm{~cm}$ f.1. Lens

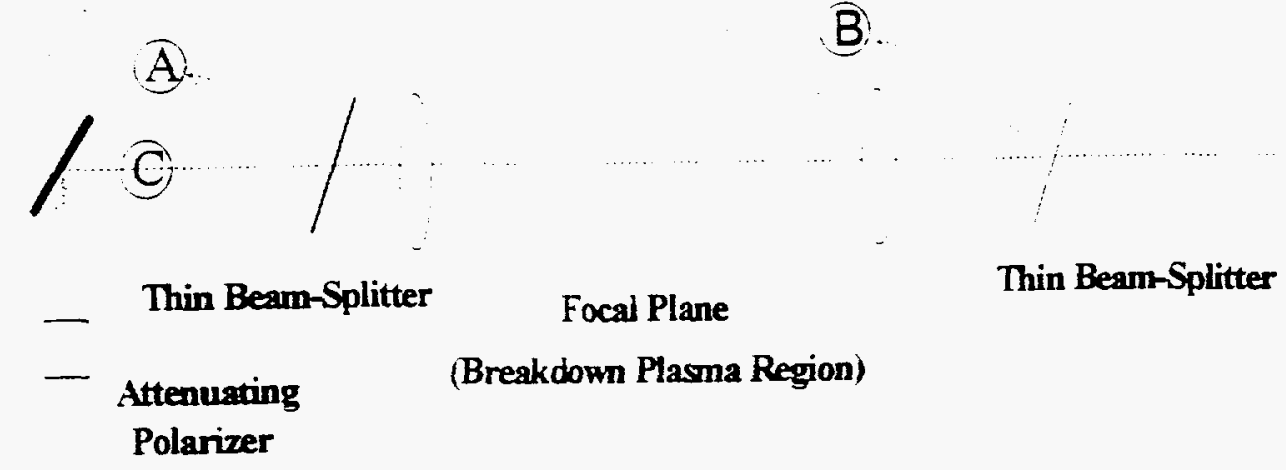

Singie or Dual Pulse Laser

Figure 12. Laser energy transmission measurement apparatus.

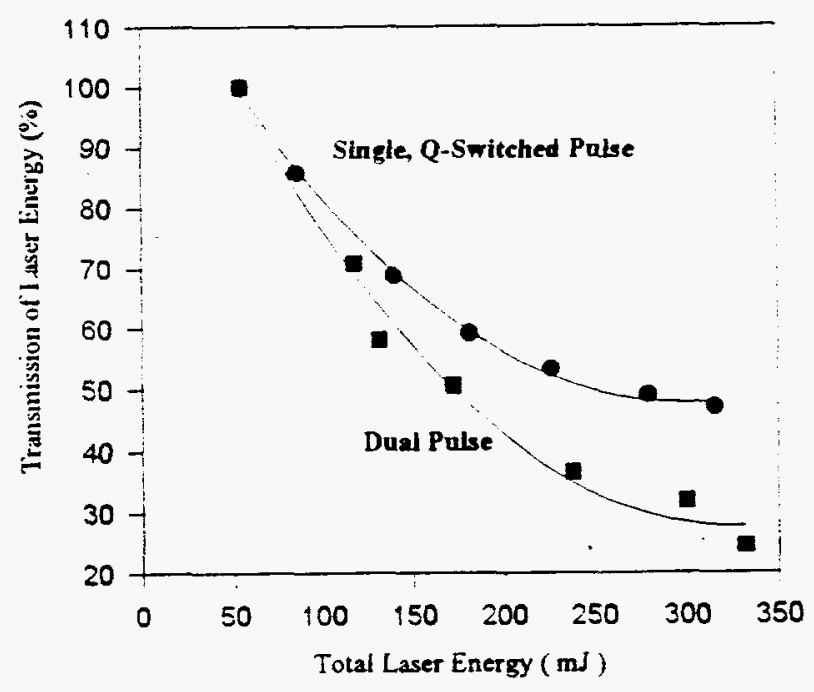

Figure 13. Laser light transmission through laser spark for single and dual laser pulse formats. 


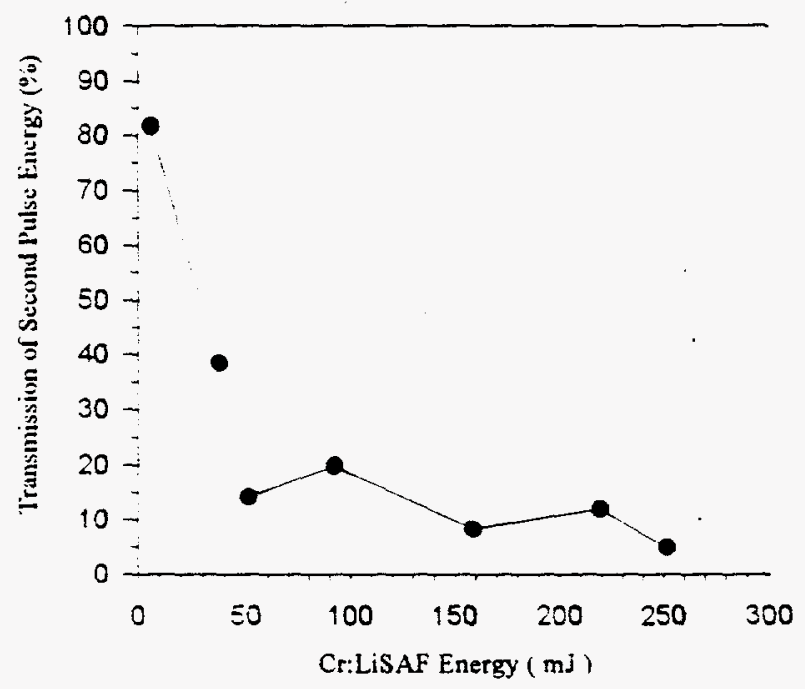

Figure 14: The transmission of second pulse energy through a laser spark as a function of second pulse energy.

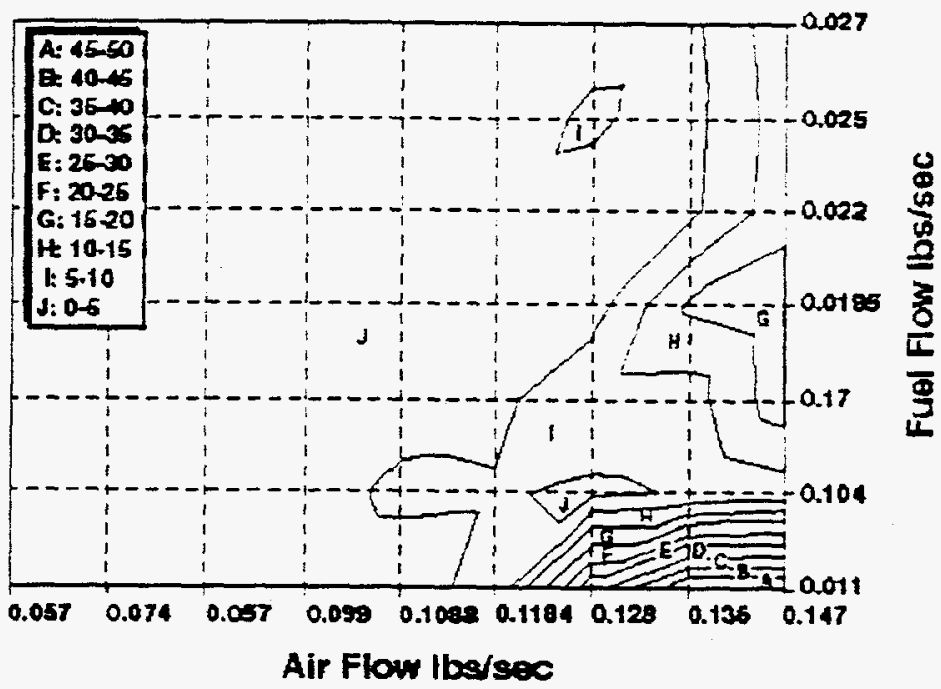

Figure 15: Allied Signal ignition results obtained at a laser energy of $123 \mathrm{~mJ}$ via a single laser pulse ignition approach. 


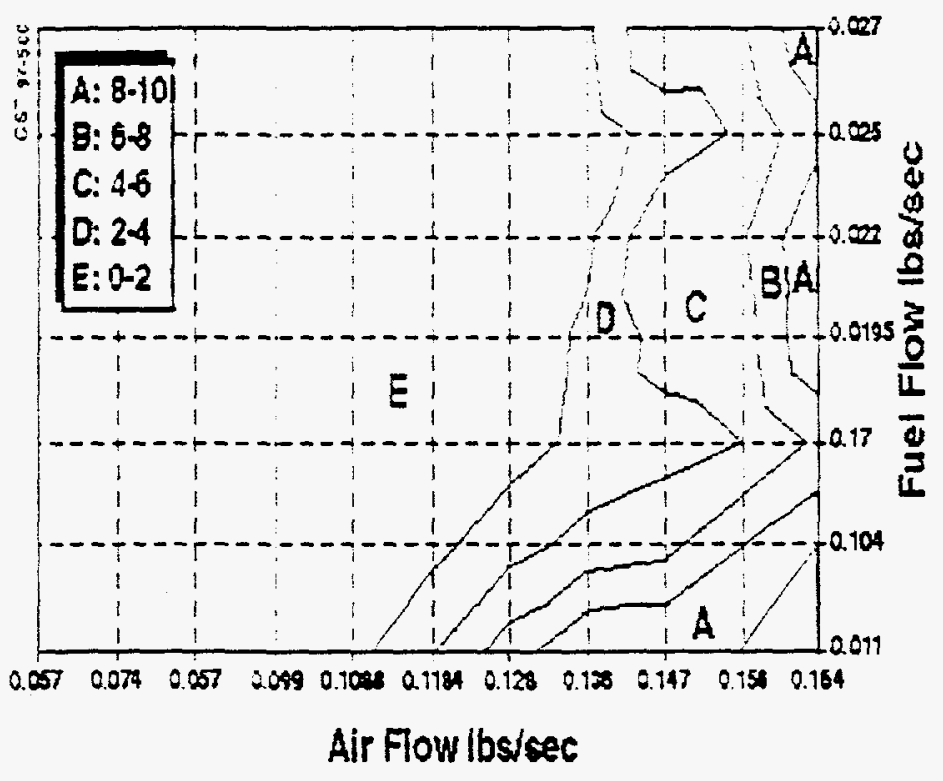

Figure 16: Dual pulse ignition results at a total laser energy of $112 \mathrm{~mJ}$.

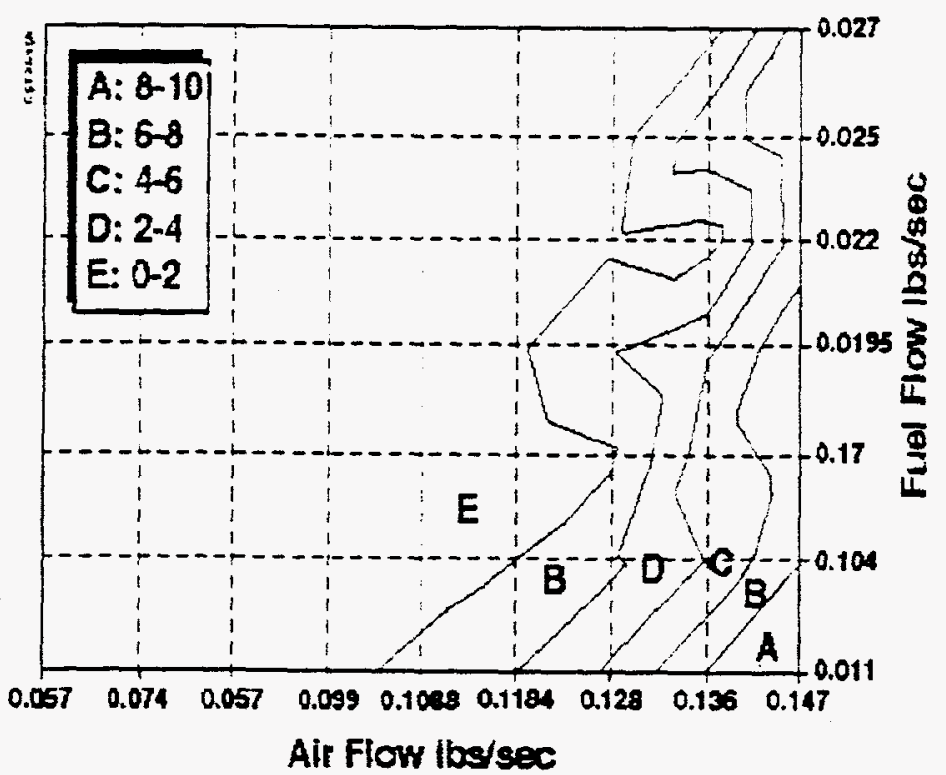

Figure 17: Dual pulse ignition results at a total laser energy of $135 \mathrm{~mJ}$. 


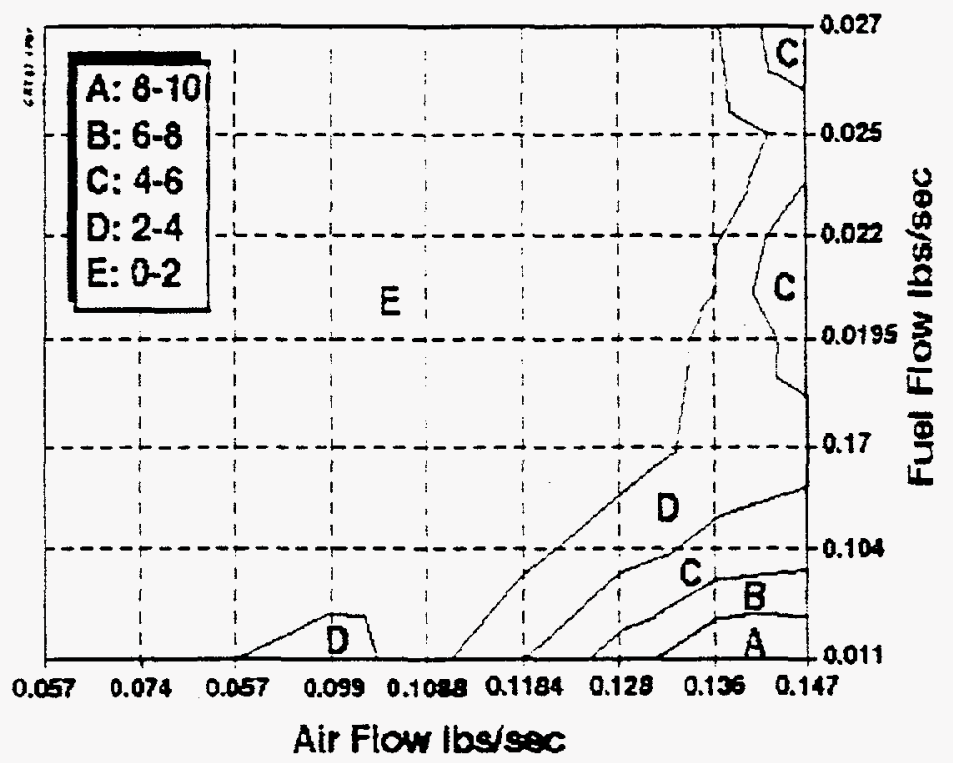

Figure 18: Dual pulse ignition results at a total laser energy of $193 \mathrm{~mJ}$.

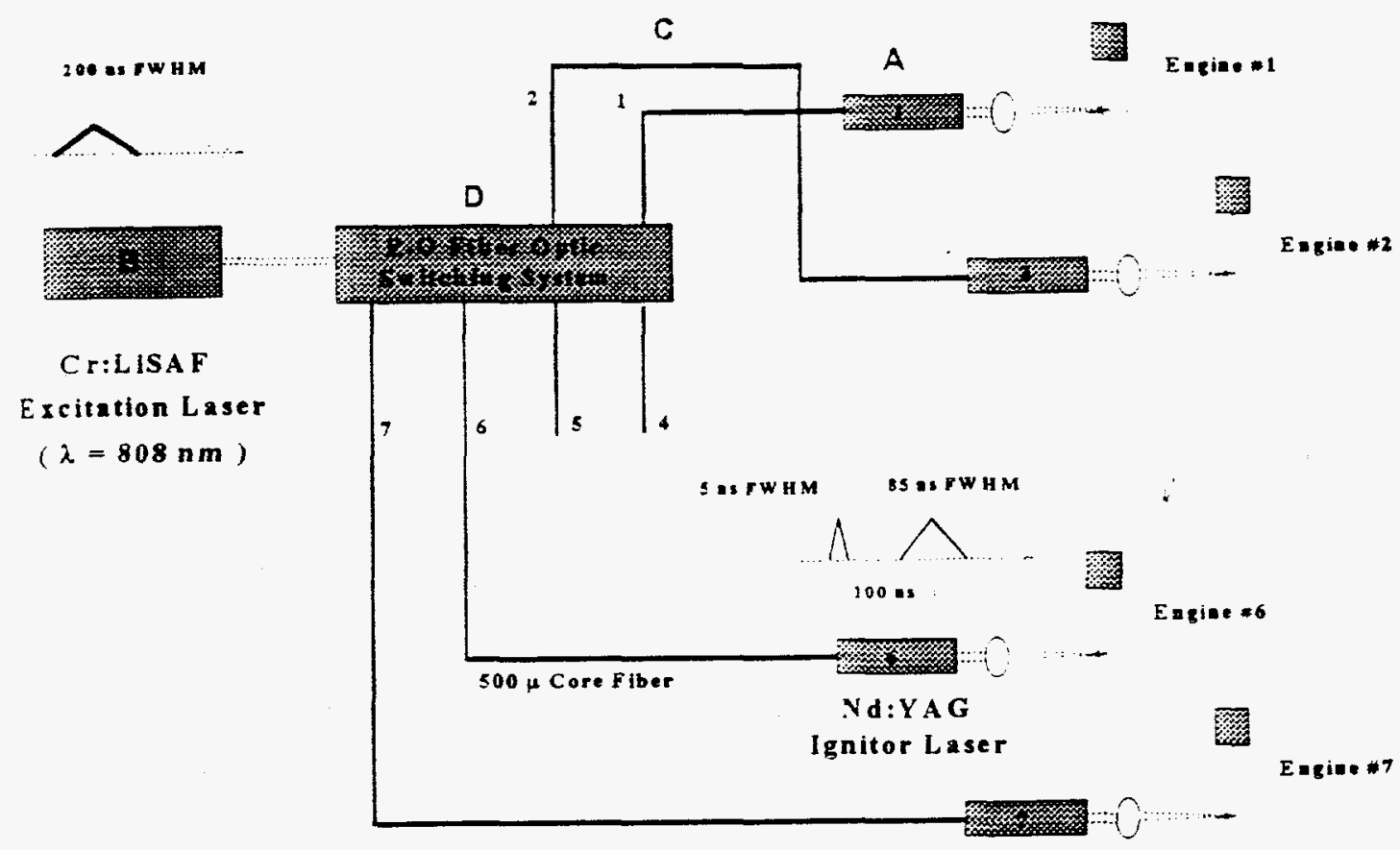

Figure 19: A compact and durable, remotely energized, dual laser pulse igniter concept. 
$-m^{2}+t^{2}=$

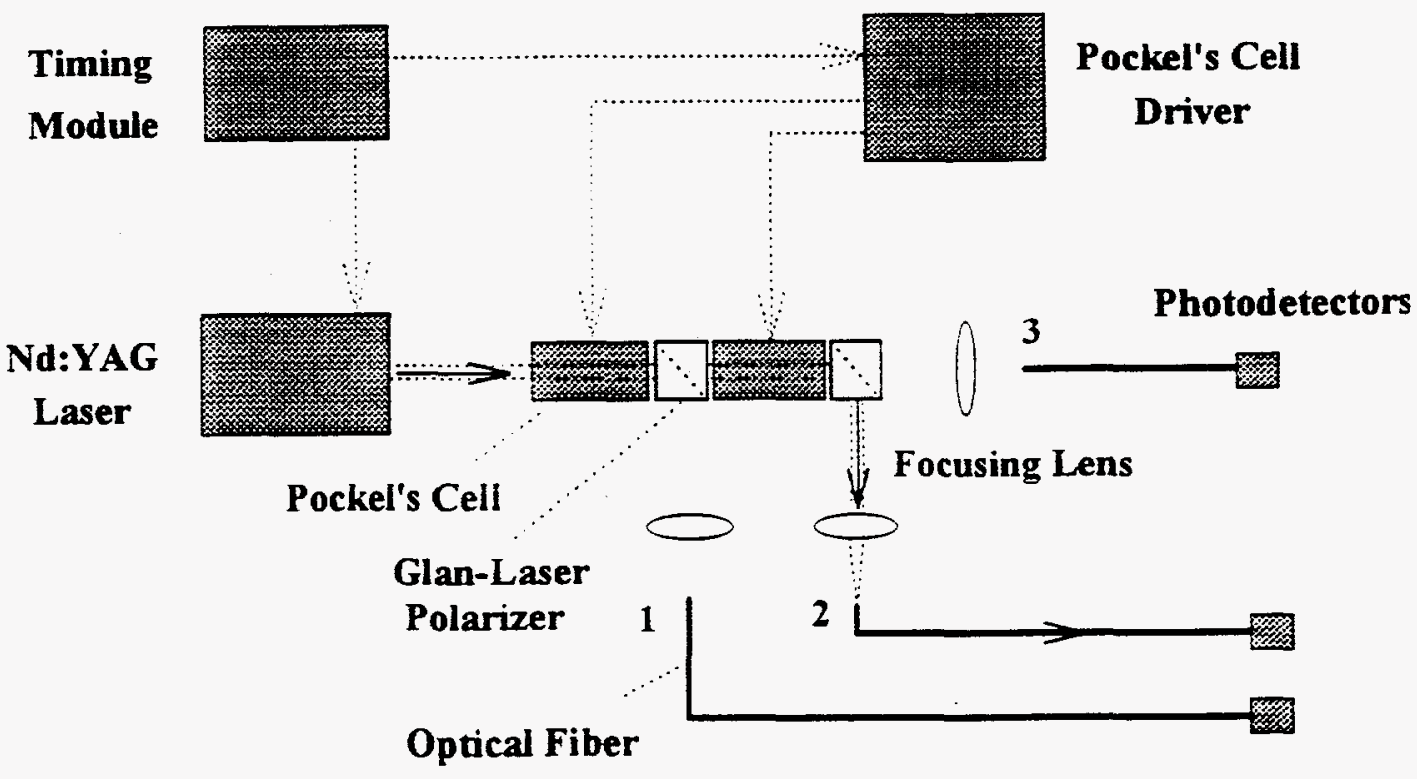

Figure 20. A prototype electro-optic fiber switching device.

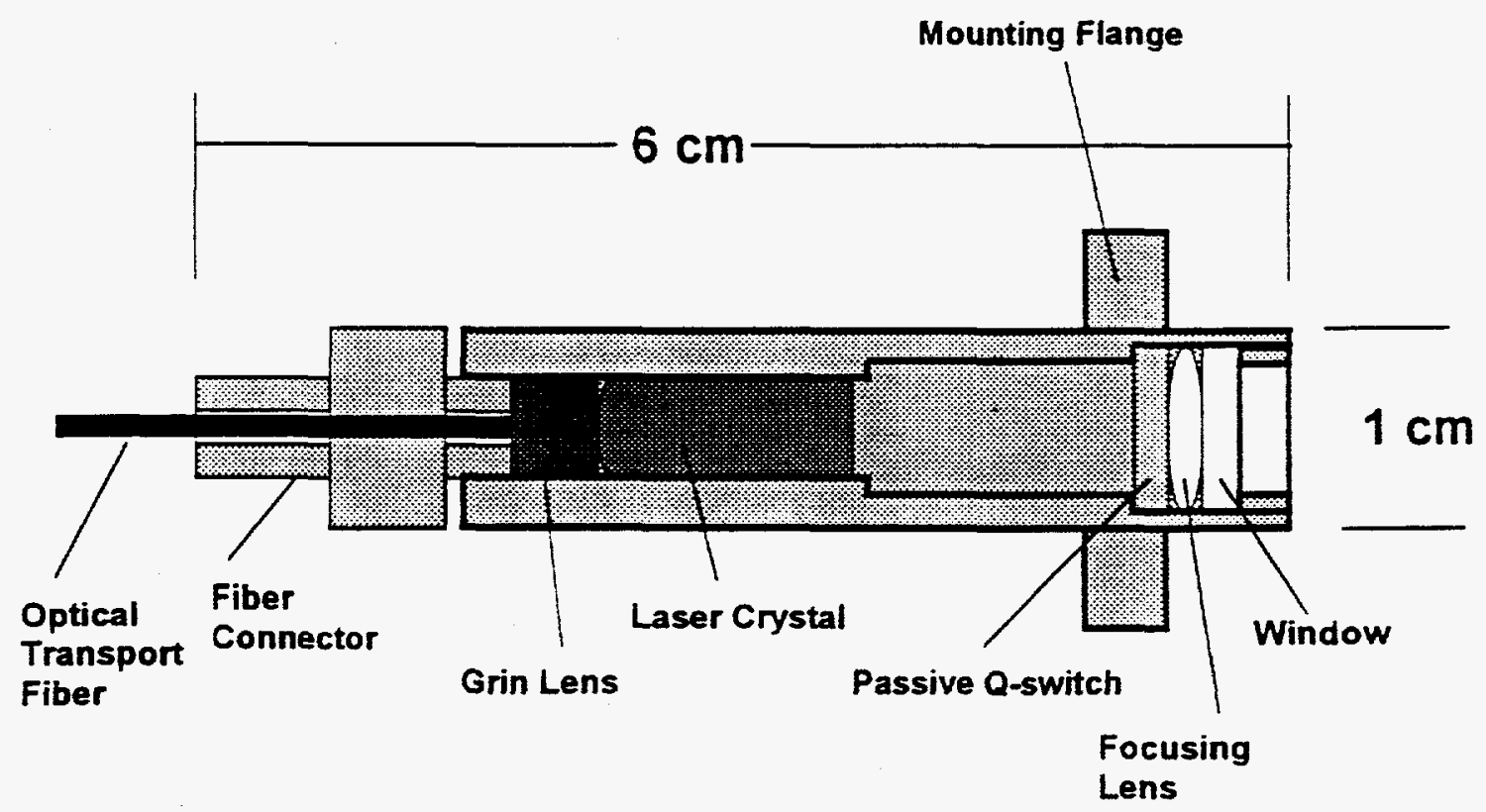

Figure 21: A compact, durable laser fuel igniter. 


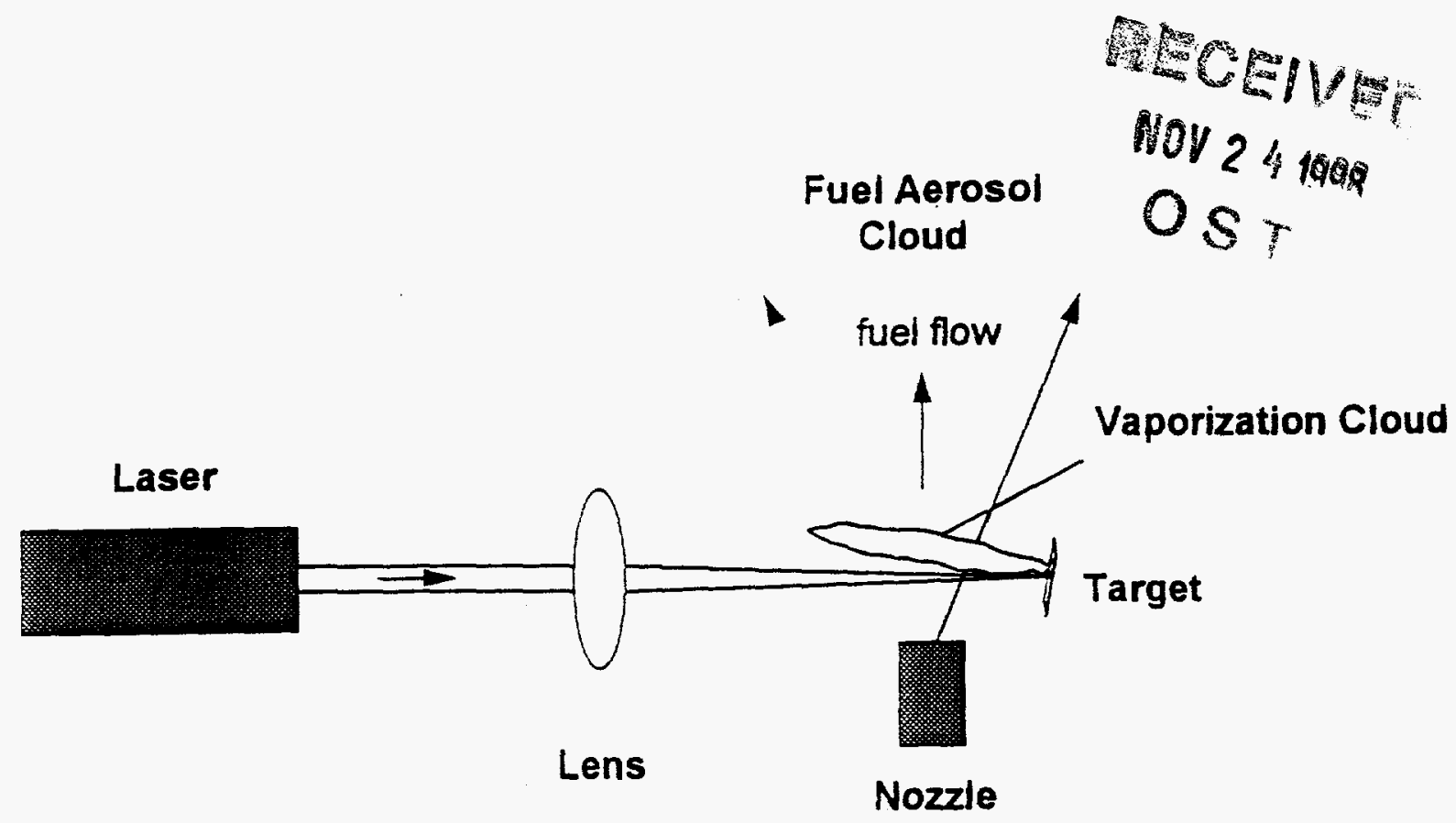

Figure 22: A laser ablation-based, fuel ignition concept. 